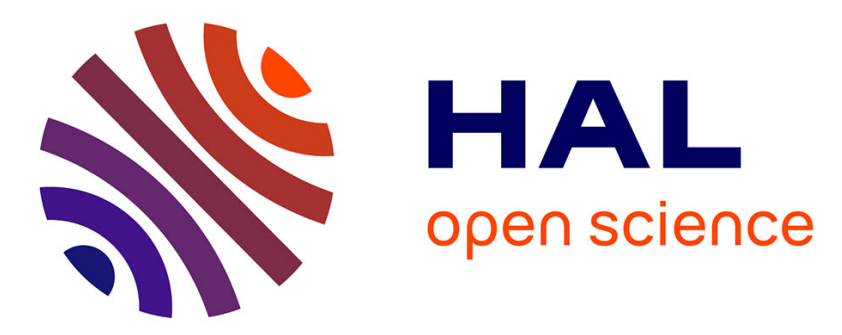

\title{
Modeling how Soluble Microbial Products (SMP) support heterotrophic bacteria in autotroph-based biofilms
}

\author{
Brian V. Merkey, Bruce E. Rittmann, David L. Chopp
}

\section{- To cite this version:}

Brian V. Merkey, Bruce E. Rittmann, David L. Chopp. Modeling how Soluble Microbial Products (SMP) support heterotrophic bacteria in autotroph-based biofilms. Journal of Theoretical Biology, 2009, 259 (4), pp.670. 10.1016/j.jtbi.2009.05.010 . hal-00554611

\author{
HAL Id: hal-00554611 \\ https://hal.science/hal-00554611
}

Submitted on 11 Jan 2011

HAL is a multi-disciplinary open access archive for the deposit and dissemination of scientific research documents, whether they are published or not. The documents may come from teaching and research institutions in France or abroad, or from public or private research centers.
L'archive ouverte pluridisciplinaire HAL, est destinée au dépôt et à la diffusion de documents scientifiques de niveau recherche, publiés ou non, émanant des établissements d'enseignement et de recherche français ou étrangers, des laboratoires publics ou privés. 


\section{Author's Accepted Manuscript}

Modeling how Soluble Microbial Products (SMP) support heterotrophic bacteria in autotroph-based biofilms

Brian V. Merkey, Bruce E. Rittmann, David L. Chopp

PII: S0022-5193(09)00237-9

DOI: doi:10.1016/j.jtbi.2009.05.010

Reference: YJTBI 5567

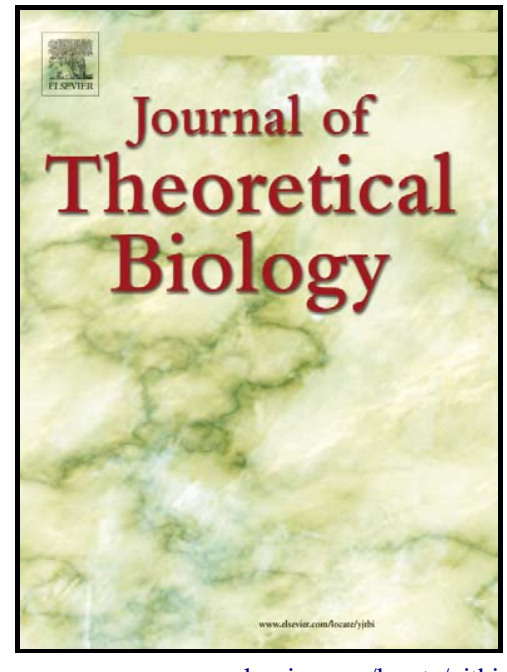

www.elsevier.com/locate/yjtb

To appear in: $\quad$ Journal of Theoretical Biology

Received date: $\quad 9$ July 2008

Revised date: $\quad 4$ April 2009

Accepted date: $\quad 18$ May 2009

Cite this article as: Brian V. Merkey, Bruce E. Rittmann and David L. Chopp, Modeling how Soluble Microbial Products (SMP) support heterotrophic bacteria in autotroph-based biofilms, Journal of Theoretical Biology, doi:10.1016/j.jtbi.2009.05.010

This is a PDF file of an unedited manuscript that has been accepted for publication. As a service to our customers we are providing this early version of the manuscript. The manuscript will undergo copyediting, typesetting, and review of the resulting galley proof before it is published in its final citable form. Please note that during the production process errors may be discovered which could affect the content, and all legal disclaimers that apply to the journal pertain. 


\title{
Modeling How Soluble Microbial Products
}

\section{(SMP) Support Heterotrophic Bacteria in}

\section{Autotroph-Based Biofilms}

\author{
Brian V. Merkey ${ }^{a, *}$ Bruce E. Rittmann ${ }^{b}$ David L. Chopp ${ }^{c}$ \\ ${ }^{a}$ Department of Environmental Engineering, \\ The Technical University of Denmark, \\ 2800 Kgs. Lyngby, Denmark \\ ${ }^{\mathrm{b}}$ Center for Environmental Biotechnology, \\ The Biodesign Institute, Arizona State University, \\ Tempe, AZ 85287-5701 \\ ${ }^{\mathrm{c}}$ Department of Engineering Sciences and Applied Mathematics, \\ Northwestern University, \\ Evanston, IL 60208
}




\section{Abstract}

Multi-species biofilm modeling has been used for many years to understand the interactions between species in different biofilm systems, but the complex symbiotic relationship between species is sometimes overlooked, because models do not always include all relevant species and components. In this paper, we develop and use a mathematical model to describe a model biofilm system that includes autotrophic and heterotrophic bacteria and the key products produced by the bacteria. The model combines the methods of earlier multi-species models with a multi-component biofilm model in order to explore the interaction between species via exchange of soluble microbial products (SMP). We show that multiple parameter sets are able to describe the findings of experimental studies, and that heterotrophs growing on autotrophically produced SMP may pursue either $r$ - or $K$-strategies to sustain themselves when SMP is their only substrate. We also show that heterotrophs can colonize some distance from the autotrophs and still be sustained by autotrophically produced SMP. This work defines the feasible range of parameters for utilization of SMP by heterotrophs and the nature of the interactions between autotrophs and heterotrophs in multi-species, multi-component biofilms.

Key words: biofilm model, multi-species, multi-component, $r$-strategist, $K$-strategist

* Corresponding Author Email address: brim@env.dtu.dk (Brian V. Merkey). 


\section{Introduction}

The surface-attached microbial communities known as biofilms are the dominant form of microbial life, with more than $90 \%$ of bacteria living in biofilm communities (Characklis and Marshall, 1990). Biofilms have a huge impact on many natural and engineered systems that support human life, in particular for applications of wastewater treatment (Rittmann, 2004; Rittmann and McCarty, 2001). For purposes of water reclamation, an important type of biofilm is the combination of autotrophic and heterotrophic bacteria (Eberl et al., 2006). Autotrophic bacteria species use an inorganic electron donor, such as $\mathrm{NH}_{4}^{+}$or $\mathrm{H}_{2}$, and inorganic carbon $\left(\mathrm{CO}_{2}\right.$ or $\left.\mathrm{HCO}_{3}^{-}\right)$as their carbon source. Due to their need to reduce inorganic carbon for synthesis, autotrophs have low true yields and maximum specific growth rates (1 per day) (Rittmann and McCarty, 2001). Conversely, heterotrophs use organic compounds as their electron donor and carbon source, and when respiring oxygen or nitrate, have high true yields and fast maximum specific growth rates (10-20 per day). Despite their differences, heterotrophs and autotrophs coexist in natural biofilms, as well as in biofilms in wastewater-treatment processes used to remove organic and ammonia pollution at the same time.

Whether or not a biofilm is multi-species, all biofilms are also inherently multi-component because they include electron donors and acceptors, active biomass, extracellular polymeric substances (EPS), residual inert material, 
and soluble microbial products (SMP) produced during normal metabolic activity (Barker and Stuckey, 1999; Laspidou and Rittmann, 2002a,b). The schematic in Figure 1 illustrates the metabolic connections between these different components of a biofilm, whose properties include the following:

- The electron donor and acceptor substrates and the carbon sources are essential components that the bacteria consume to gain energy and grow.

- Continuous decay of active bacteria produces residual, inert biomass that accumulates as a solid.

- Normal metabolic activity releases extracellular polymeric substances (EPS), a solid that acts as a "glue" to hold the biofilm together and to the surface.

- Normal metabolic activity and hydrolysis of EPS release organic soluble microbial products (SMP) that are available to heterotrophs as an electron donor and carbon source; when the SMP comes from autotrophs, it augments the organic material available to the heterotrophs.

These components all play a role in the growth and evolution of a biofilm community. EPS help maintain the integrity of the biofilm structure, but EPS production diverts electrons from biomass growth which may affect long-term species success (Kreft and Wimpenny, 2001; Xavier and Foster, 2007). SMP capture the natural products of bacterial growth (the utilzation-associated products, UAP) and hydrolysis (the biomass-associated products, BAP) and allows for an interaction between bacterial species (Barker and Stuckey, 1999). The possibility for interactions between heterotrophs and autotrophs expand 
when the multiple components are included, and in an autotrophically based system, the existence of the heterotrophs depends on autotrophic production of EPS and SMP (Bishop et al., 1995; Furumai and Rittmann, 1992, 1994; Kindaichi et al., 2004; Okabe et al., 1996; Rittmann et al., 2002; Zhang et al., 1994). Zhang et al. (1994) in particular expressed the need for a multi-species biofilm model that tracks these by-products in addition to the bulk substrates, and it is this need that we address here.

The ability to closely model the growth of a multi-species, multi-component biofilm is hampered, though, because the parameters that describe SMP and EPS production and SMP consumption are not well-established (Barker and Stuckey, 1999). Reasonable parameter values for SMP utilization have been given by Laspidou and Rittmann (2002a) and Noguera et al. (1994), but these parameters were calculated for a single-species Pseudomonas atlantica system, and, as such, are not necessarily applicable to all bacterial species or biofilm systems. In particular, it is not known which type of growth strategy heterotrophs growing on the limited resource of autotrophically produced SMP tend to pursue: an $r$ - or $K$-strategy (Andrews and Harris, 1986; Rittmann and McCarty, 2001; Velicer et al., 1999) or even a yield-strategy (Kreft, 2004; Kreft and Bonhoeffer, 2005). A heterotroph species that is an $r$-strategist has a high maximum specific growth rate and would be capable of fast growth on a relatively high concentration of SMP, but this growth could be impaired under low SMP concentrations. On the other hand, a heterotroph species that 
is a $K$-strategist would be able to scavenge low SMP concentrations, but its lower maximum specific growth rate could cause it to be out-competed by the autotrophs for space or oxygen. Alternatively, a yield-strategy approach would mean efficient conversion of SMP to biomass by the heterotrophs, but again a low specific growth rate could potentially lead to the heterotrophs being out-competed. It is likely that different heterotrophic species pursue different strategies, but it is not immediately clear which strategy provides the best fit with experimental findings, or whether growth on SMP is only possible with one of these strategies.

The objective of the work presented here is to use a multi-species, multicomponent biofilm model to quantify and evaluate the importance of including SMP when modeling biofilm systems and, in particular, to explore the effects of SMP-parameter values on the ability of heterotrophs to subsist solely on SMP. Multi-species biofilm models have previously been applied to study many different systems (Alpkvist and Klapper, 2007b; Kissel et al., 1984; Rauch et al., 1999; Rittmann and Manem, 1992; Stoodley et al., 2002b; Wanner and Gujer, 1986; Wanner and Reichert, 1995), and previous work in multicomponent biofilm models includes Laspidou and Rittmann (2004a,b), Furumai and Rittmann (1992), Furumai and Rittmann (1994), Rittmann et al. (2002), and Wood and Whitaker (2000). Combining the multi-species and multi-component approaches allows us to investigate systems not addressable by previous models: for example, neither the early multi-species model of 
Wanner and Gujer (1986) nor the Unified Model of Laspidou and Rittmann (2004a,b) is useful for modeling a system where the heterotrophs subsist entirely on organic carbon in the form of SMP produced by the autotrophs; the combined model we present here overcomes this limitation. In order to explore these complex biofilms, we began with a multi-dimensional biofilm model similar to that of Alpkvist and Klapper (2007b), who described the benefits of a continuum model for biofilm growth over cellular automata or individualbased models. Expanding this model to include multiple components as well as multiple species, we explored the dependence of multi-species coexistence on SMP and EPS.

\section{Modeling Approach}

This model includes three distinct regions within the computational domain, $\Omega$, as shown in Figure 2: the biofilm matrix, $\Omega_{B}$, where the solid components grow and decay and where the dissolved components are produced or consumed; the bulk fluid, $\Omega_{F}$, inside which the dissolved components are kept at a fixed concentration; and, between these two regions, a diffusion layer, $\Omega_{L}$, where the dissolved components diffuse, but are neither produced nor consumed. Also shown in Figure 2 is the biofilm-liquid interface, $\Gamma$. 


\subsection{Variables in the Model}

The model tracks the growth of four different types of biomass: active biomass in the form of autotrophic and heterotrophic bacteria $\left(X_{a}\right.$ and $\left.X_{h}\right)$, inactive biomass $\left(X_{i}\right)$, and extracellular polymeric substances $\left(X_{e}\right)$. Each biomass component is represented in the model by means of a maximum density, $\rho_{j}$ (different for each species), and a volume fraction, $X_{j}$. Mass conservation requires that the total volume fraction,

$$
X_{a}+X_{h}+X_{i}+X_{e}=1
$$

remain constant in both time and space. Each species grows in proportion to its mass, $\rho_{j} X_{j}$.

Following the Unified Model of Laspidou and Rittmann (2002a,b), the model also tracks five different types of soluble species: $S_{a}$ is the exogenous electron donor for the autotrophs; $S_{h}$ is the exogenous electron donor for the heterotrophs; UAP are utilization-associated products that are released as a normal part of substrate utilization by autotrophs and heterotrophs and also serve as an internal electron donor for the heterotrophs; BAP are biomassassociated products that are produced by hydrolysis of EPS and serve as a second internal electron donor for the heterotrophs; and $A$, the common electron acceptor for both active species. Note that the term soluble microbial products (SMP) refers to the two organically produced electron donors UAP 
and BAP.

\subsection{Dissolved-Phase Equations}

Table I describes the processes that affect the dissolved-component concentrations inside the biofilm, with parameter definitions and example values given in Tables II and III. Each rate is based on multiplicative Monod kinetics, where the $\hat{q}_{j}$ terms are the maximum specific utilization rates for each process, and $K_{j}$ is the half-maximum-rate concentration of the corresponding component. The net reaction rate $\eta_{j}$ for each component may be found by multiplying each coefficient times the corresponding rate and summing down the corresponding column; a value of $\eta_{j}$ greater than zero corresponds to net production of a component, while a negative value of $\eta_{j}$ corresponds to net consumption of the component. For example, the net reaction rate for BAP is given by:

$$
\eta_{\mathrm{BAP}}=-r_{\mathrm{BAP}} \rho_{h} X_{h}+k_{h y d} \rho_{e} X_{e}
$$

where the reaction rate $r_{\mathrm{BAP}}$ is given in the caption to Table I.

Electron donors $S_{a}$ and $S_{h}$ are consumed by bacteria in the biofilm, and consumption requires the electron acceptor, $A$. The consumption process produces UAP; these UAP are metabolized by the heterotrophs (again in the presence of the electron acceptor) with a corresponding rate. The heterotrophs also consume BAP, which are produced from EPS via hydrolysis. Note that, following 
Laspidou and Rittmann (2002a,b), SMP consumption by the heterotrophs does not result in further SMP or EPS production; this is done to prevent unlimited cycling of electrons. Finally, the electron acceptor, $A$, is also consumed during biomass respiration, which serves as an additional energy source.

The dissolved components are diffusion-limited and assumed to be in a quasisteady state at all times, since biofilm growth is very slow compared to diffusion rates (Kissel et al., 1984). Thus, the steady-state mass-balance equations for these components for any location inside the biofilm take the form:

$$
0=D_{j} \nabla^{2} S_{j}+\eta_{j}
$$

where $\eta_{j}$ is the net reaction rate of substrate $S_{j}$, given by adding all columnar terms in Table I, and $D_{j}$ is the local diffusion coefficient. Inside the biofilm, $D_{j}$ is decreased by a factor 0.8 from its value in water to account for the mass transfer resistance of the biofilm (Rittmann and Manem, 1992; Rittmann and McCarty, 2001). Outside the biofilm, in the diffusion layer between the biofilm and bulk liquid, the reaction term $\eta_{j}$ is zero because the dissolved components merely diffuse through the water with no reaction occuring in that region.

\subsection{Solid-Phase Equations}

Table I describes how different processes affect biomass growth. All rates are normalized by the density, $\rho_{j}$, of the species of interest, which is assumed 
constant. Biomass increases by the metabolism of the dissolved components, with the autotrophs metabolizing $S_{a}$, and the heterotrophs metabolizing $S_{h}$, $\mathrm{UAP}$, and BAP. The active biomass is consumed via respiration and decay processes, the former consuming $A$ to produce energy, and the latter producing residual inert biomass. EPS are produced during $S_{a}$ and $S_{h}$ metabolism and are transformed into BAP by hydrolysis.

The solid components grow in time and move by advection, and each has a mass-balance equation of the form:

$$
\frac{\partial X_{j}}{\partial t}+\nabla \cdot\left(\vec{u} X_{j}\right)=\mu_{j}
$$

The left side of each equation describes the conservation of mass of each species and depends on the biomass velocity $\vec{u}$. The term on the right side describes the net growth of each solid component, and the growth terms $\mu_{j}$ are found by summing down the columns in Table I just as for the dissolved-component reaction rate $\eta_{j}$. The growth terms $\mu_{j}$ use Monod kinetics to capture the behavior of different species, just as for the dissolved components; the same $\hat{q}_{j}$ and $K_{j}$ parameters appear in the biomass growth terms as in the dissolved component reaction rates $\eta_{j}$. For the solid components, species that are $r$ strategists will tend to have high values for both $\hat{q}_{j}$ and $K_{j}$, while for $K$ strategist species these values will be lower.

The biomass velocity, $\vec{u}$, required to track biomass advection, is found by 
summing the mass-balance equations of the four types of biomass. Then, using (1) we find that:

$$
\nabla \cdot \vec{u}=\sum_{j} \mu_{j}
$$

We assume potential flow for the velocity because the advective velocity is smooth and irrotational (Klapper et al., 2002; Stoodley et al., 2002a). We may then write the velocity as the gradient of a scalar function: $\vec{u}=\nabla \Phi$. Substituting this expression in (4) yields in an elliptic equation for the potential:

$$
\nabla^{2} \Phi=\sum_{j} \mu_{j}
$$

which must be solved in $\Omega_{B}$. This method of computing the advective veloc-

ity is analogous to Dockery and Klapper (2001) and Alpkvist and Klapper (2007b), where they refer to the potential as 'pressure.'

\subsection{Moving Interface}

Modeling the evolution of a biofilm involves letting the biofilm-fluid interface move in time, which we accomplish using the level set method (Osher and Sethian, 1988; Sethian, 1999). This approach, or a slight variation, has been used by Dockery and Klapper (2001), Xavier et al. (2005), Alpkvist et al. (2006), and Duddu et al. (2007). In the level set method, the interface is represented by the zero-level contour of a higher-dimensional function $\phi$, which is often chosen to be the signed distance from the interface. The interface is 
evolved by means of the following equation:

$$
\phi_{t}+F\|\nabla \phi\|=0
$$

where $F$ is the normal velocity of the interface. We find the normal velocity from the gradient of the biomass velocity potential, $\vec{u}=\nabla \Phi$. The normal velocity of the biofilm interface, $\Gamma$, is the sum of the velocity due to growth and decay, $\nabla \Phi$, and the rate of surface erosion, $\sigma$ :

$$
F=\frac{\partial \Phi}{\partial n}+\sigma
$$

We use the height-based detachment model, $\sigma=-k_{d e t} y^{2}$, as in Wanner and Gujer (1986) and Xavier et al. (2005). Other forms of detachment, as in Horn et al. (2003), Morgenroth and Wilderer (2000), or Duddu et al. (2008), may be easily added by using a different form for $\sigma$.

Our approach to capturing erosion effects is somewhat ad hoc, but it serves the purpose of imposing some limit on growth in order to keep the biofilm region within the computational domain. Previous studies by Picioreanu et al. (1999), Alpkvist and Klapper (2007a), and Duddu et al. (2008) have shown the benefit of including the fluid-structure interaction between the biofilm and the surrounding fluid, which is an improvement over the simple model used here. However, in this work we are primarily concerned with dynamics within the biofilm, so we are more interested in capturing the effects of erosion (limited 
growth height) rather than the cause of erosion (fluid-structure interactions).

\subsection{Boundary Conditions}

The boundary conditions for each set of equations are governed by the type of interfaces at each boundary. In this system, we assume that the attachment surface is impermeable, and so the boundary condition for the dissolved components (here written for $S_{a}$ ) is a zero-flux condition:

$$
\frac{\partial S_{a}}{\partial n}=0
$$

The boundary condition for the other solutes is identical.

At the biofilm-liquid interface, $\Gamma$, we assume continuous substrate concentration and conservation of flux. These two boundary conditions written for $S_{a}$ are:

$$
\left[S_{a}\right]=0, \quad\left[D_{S_{a}} \frac{\partial S_{a}}{\partial n}\right]=0
$$

where the quantities in brackets indicate the difference in the argument across the boundary. Here, $D_{S_{a}}$ is the diffusion coefficient of $S_{a}$ in water. Inside the biofilm we multiply the diffusion coefficients by 0.8 to account for the increased mass-transport resistance (Rittmann and McCarty, 2001). These boundary conditions are solved numerically using the Immersed Interface Method (IIM) (Leveque and Li, 1994), which modifies the default 5-point diffusion stencil 
near the interface to account for the boundary conditions. The IIM ensures a globally second-order solution to the elliptic equations. We also assume a diffusion layer between the bulk fluid and the biofilm surface; in this layer the dissolved components diffuse but are neither consumed nor produced. This is a phenomenon observed by, for example, Zhang et al. (1994).

Biomass has a no-flux boundary condition at the substratum; hence the velocity potential, $\Phi$, has a Neumann boundary condition at that interface:

$$
\frac{\partial \Phi}{\partial n}=0
$$

As in Dockery and Klapper (2001) and Alpkvist et al. (2006), $\Phi$ is held constant at zero at the biofilm-liquid interface. The detachment boundary condition at the surface is taken care of by (7).

\subsection{Numerical Solution}

To solve the PDE system, we discretize in space and time, treating each region of the domain differently (Figure 2). The biomass equations (3) and velocity equation (5) are solved only within the biofilm region, $\Omega_{B}$, whereas the dissolved components (2) are solved in the biofilm and in the diffusion layer, $\Omega_{B} \cup \Omega_{L}$. With the region so divided, we proceed as follows:

1. Solve the dissolved-phase equations (2) simultaneously using the Newton- 
Raphson method. In this method, the solution to the linear system

$$
M \vec{S}=\vec{f}(\vec{S})
$$

is found iteratively via

$$
\vec{S}^{n+1}=\vec{S}^{n}-\vec{\delta}
$$

where $\vec{\delta}$ is the solution to the Jacobian system $J \vec{\delta}=\vec{H}, J$ is computed from the linear operator, $M$, and $\vec{H}$ is computed from the original equations (2).

2. Calculate the biomass velocity in $\Omega_{B}$ using (5) and take the gradient of the resulting potential.

3. Update the solid-phase components individually in $\Omega_{B}$ using a conservative, upwinding discretization of equations (3).

4. Update the biofilm interface location, $\Gamma$, using (6).

We repeat this procedure for each time step in order to evolve through time.

\subsection{Modeling Strategy}

Because the parameters affecting SMP utilization by the heterotrophs $\left(\hat{q}_{\mathrm{UAP}}\right.$, $\hat{q}_{\mathrm{BAP}}, K_{\mathrm{UAP}}$, and $\left.K_{\mathrm{BAP}}\right)$ are not well-studied experimentally, we undertook a strategy to vary these parameters over a range of reasonable values in order to determine which ranges yield biofilm growth that mirrors experimental 
findings. The parameter values for SMP utilization given by Laspidou and Rittmann (2002a) and Noguera et al. (1994), while determined for a specific system, provide a place to begin when considering variations in these parameters. As another guide to studying SMP use by heterotrophs, we consider the optimal growth of heterotrophs using bulk-liquid COD with parameters from Wanner and Gujer (1986). In this work we limit the parameter exploration to variations in parameters describing SMP utilization by the heterotrophs and for now do not consider variations in EPS production or hydrolysis.

In order to isolate the effect of heterotrophic growth on SMP, we set the concentration of heterotroph electron donor $S_{h}$ to zero in the bulk (Table II), so that UAP and BAP are the only electron donors available for heterotrophic growth (though in general this model is equally applicable to systems with nonzero bulk COD). Following Laspidou and Rittmann (2002a,b), SMP utilization by the heterotrophs does not yield further SMP or EPS production, and so in this system the autotrophs are the sole source for SMP within the biofilm; any growth by the heterotrophs therefore depends on autotrophic production of SMP.

In all studies, the model was initialized, as in Alpkvist and Klapper (2007b), with a random distribution of biomass that was then smoothed with a Gaussian convolution; the total initial volume fraction was split equally between autotrophs, heterotrophs, and inert biomass, as in Table II. For the system considered in this study, the rough initial biofilm surface evolves to a flat 
steady-state profile for two reasons: (1) the slow-growing autotrophs initially dominate the biofilm, and because of their low growth rate they tend to grow flat (Picioreanu et al., 1998), and (2) the faster-growing heterotrophs, while more plentiful at later times, receive their electron donor solely from within the biofilm so there is no opportunity for a fingering instability to arise, as discussed in Picioreanu et al. (1998) and Dockery and Klapper (2001).

Kindaichi et al. (2004) reported that, in an autotrophically based system, the ratio of heterotrophs to autotrophs after three months of evolution was roughly 1:1; this fits with the conclusions of earlier work such as Zhang et al. (1994), and we use this result as a measure for which sets of parameters yield physically-meaningful results. Following the three months measure, we run our simulations for 90 days. After determining some reasonable parameter sets that yield a roughly 1:1 species balance, we proceed to study some of the effects of inoculation on the resulting multi-species biofilm.

\section{Results and Discussion}

\subsection{Relative Importance of UAP and BAP}

Given that we have included both types of SMP in the model, we evaluated the relative importance of UAP and BAP to heterotrophic growth by first removing heterotrophic growth on first one and then the other SMP component; this 
was done by setting either $\hat{q}_{\mathrm{UAP}}$ or $\hat{q}_{\mathrm{BAP}}$ to zero, with the other SMP parameters as given in the LR-1 case in Table $\mathrm{V}$. The relative importance of these two components on heterotrophic growth is summarized in Figure 3. Plotted in Figure 3 is the relative fraction of heterotrophs to autotrophs $\left(X_{h} / X_{a}\right)$ within the biofilm as a function of time for each of the cases of SMP utilization. It is easily seen in the plot that heterotrophic growth occurs primarily via metabolism of UAP and very little via BAP metabolism. This trend is not too surprising given that the UAP utilization rate is much larger than the BAP utilization rate (more than a factor of 18 from Laspidou and Rittmann (2002a), Table III). Also at play are the different sources of UAP and BAP within the biofilm: UAP is produced continuously during autotrophic growth (at a maximum specific rate of $\hat{q}_{S_{a}} \cdot Y_{S_{a}}=0.95 d^{-1}$ ), whereas BAP is only produced via hydrolysis of EPS (which occurs at a slower specific rate of $0.17 d^{-1}$. Later we will use this result in summarizing heterotrophic growth on SMP by focusing on UAP parameters.

\section{2 $\hat{q}$ Variable, Very Low K (K-strategy)}

The previous section underscores the strong effect utilization rates may have on the resulting heterotroph-to-autotroph fraction; hence, we explored the influence of $\hat{q}_{\mathrm{UAP}}$ and $\hat{q}_{\mathrm{BAP}}$ values on the resultant biofilm composition. To do this, we set the $K$ values very low so that substrate limitation was not a factor; this corresponds to a $K$-strategist behavior. We used $K_{\mathrm{UAP}}=0.01 \mathrm{mg} \mathrm{COD} / L$ 
and $K_{\mathrm{BAP}}=0.0085 \mathrm{mg} \mathrm{COD} / L$, four orders of magnitude smaller than the values used in Laspidou and Rittmann (2002a) and low enough that for all but the lowest SMP concentrations the net growth rate did not vary. We kept the ratio of $\hat{q}_{\mathrm{UAP}}$ to $\hat{q}_{\mathrm{BAP}}$ equal to the ratio of these values in Laspidou and Rittmann (2002a); the values used are given in Table IV. We based the variations on the value of $\hat{q}_{\mathrm{UAP}}$, which spanned the range from the Laspidou and Rittmann (2002a) SMP-utilization rate to the Wanner and Gujer (1986) COD-utilization rate.

Figure 4 shows the relative fraction of heterotrophs to autotrophs $\left(X_{h} / X_{a}\right)$ in the biofilm as a function of time for these different parameter sets. The relative fraction drops quite quickly early in the simulation, but the heterotrophs recover soon after; this drop and recovery are a result of the delay between SMP production by the autotrophs and its utilization leading to growth by the heterotrophs. The curves show a recovery from the drop that is proportional to the utilization rate. From the plot we also see that, when $K$ is not a factor, any of the $\hat{q}$ values is sufficient to yield heterotroph subsistence on SMP; furthermore, all $\hat{q}$ values eventually lead to the relative fraction of heterotrophs in the biofilm being greater than 1 . This conclusion underscores the ability of $K$-strategists to survive on low substrate concentrations, as is the case for subsistence on SMP in this multi-species biofilm.

Note also that after roughly 40 days the ratio of heterotrophs to autotrophs is roughly constant, meaning that the species balance has reached a steady state. 
This is due to the balance of SMP production and consumption potentials of the autotrophs and heterotrophs, respectively. More will be said about this plateau in later sections.

\subsection{K Variable, $\hat{q}$ From Laspidou and Rittmann (2002a)}

Having seen that a $K$-strategist approach allows the heterotrophs to subsist easily within the biofilm, we explored the effects of varying $K$ for a fixed utilization rate. For the first tests, we used the SMP-utilization rates from Laspidou and Rittmann (2002a) (LR), which are given in Table III. We then modified the $K_{\mathrm{UAP}}$ and $K_{\mathrm{BAP}}$ values from their values in Laspidou and Rittmann (2002a), also given in Table III. The $K$-values were modified using a scaling factor $\alpha$, with $K_{\text {used }}=\alpha K_{L R}$ for UAP and BAP, with $\alpha$ varied over 4 orders of magnitude, as shown in the legends in Figure 5. Values of $\alpha>1$ were not considered, because the reference values from Laspidou and Rittmann (2002a) already showed evidence of being high.

Figure 5(a) shows the relative fraction of heterotrophs to autotrophs as a function of time for these varied- $K$ experiments. This plot shows the same initial drop in ratio at the beginning, but then a recovery for only some of the parameter sets. This shows that, for these utilization rates, only the low $K$ values (low $\alpha$ ) allow the heterotrophs to subsist and yield an autotroph-toheterotroph ratio in line with the results of Kindaichi et al. (2004), further sup- 
porting the conclusion of the previous section that $K$-strategist heterotrophs are well-suited to subsistence on SMP.

\subsection{K Variable, $\hat{q}$ From COD Utilization (Wanner and Gujer, 1986) (r- strategy)}

Similar to the previous section, $K$ values were again varied for a fixed $\hat{q}$, but here $\hat{q}_{\mathrm{UAP}}$ was set equal to the COD utilization rate from Wanner and Gujer (1986): $\hat{q}_{\mathrm{UAP}}=12.1 \mathrm{mg} \mathrm{COD} / \mathrm{mg} \mathrm{COD} / \mathrm{d}$. The value for $\hat{q}_{\mathrm{BAP}}$ was kept in the same ratio to $\hat{q}_{\mathrm{UAP}}$ as in the previous case, so that $\hat{q}_{\mathrm{BAP}}=$ $0.67 m g \mathrm{COD} / m g \mathrm{COD} / d$.

Figure 5(b) shows the relative fraction of heterotrophs to autotrophs as a function of time, and a comparison with Figure 5(a) shows that, for these higher utilization rates, a larger $K$ value (larger $\alpha$ ) is sufficient to yield coexistence of species; this is a result of the higher utilization rate ( $r$-strategy) overcoming the effects of a high $K$. For an $r$-strategist, growth at the higher rate lowers the importance of SMP concentration in determining the net reaction rate (Velicer et al., 1999), which is why many potential values for $K$ are able to yield heterotroph subsistence.

The effects of variations in the scaling parameter $\alpha$ are also shown in Figure 6, where the amounts of the autotrophs and heterotrophs are plotted over time. These amounts were calculated by multiplying the volume fraction of each 
species by the total biofilm area at each point in time. One may see that the autotrophs are only tangentially affected by the parameter variations, with a 2orders-of-magnitude variation in $\alpha$ yielding a roughly $25 \%$ change in long-term autotroph presence. The change in autotroph amount is due to the increased competition with the heterotrophs for space and electron acceptor. This is easily seen by looking at the early evolution of autotroph amount: for larger $\alpha$ (meaning larger $K$ and therefore slower heterotroph growth on SMP) the curves of autotroph amount track one another and do not diverge until later times, while, for the smallest $\alpha$ (smaller $K$ so high heterotroph scavenging), the autotroph amount does not rise as high in the early time due to earlier competition with fast-growing heterotrophs.

The effect of different scaling values on the heterotroph amount is very dramatic and is also seen in Figure 6. For the lowest $\alpha$ values, the heterotrophs quickly reach a steady-state amount that is quite low, but for the highest values the heterotroph amount grows steadily. The middle values of $\alpha$ yields a variation over a much longer time scale, with the heterotrophs in these cases again tending toward a high presence within the biofilm.

The difference in amounts of autotrophs and heterotrophs for these cases may be understood more clearly by looking at the rates of UAP production and consumption, shown in Figure 7. The production of UAP by the autotrophs follows the same trend of autotroph amount in Figure 6, with a roughly $20 \%$ variation in the rate of UAP production due to varying $K$ values for the 
heterotrophs. The UAP consumption rate explains the large difference in heterotroph amounts seen in Figure 6: the large value of $\alpha=0.03$ yields a rate of nearly zero, resulting in a low heterotroph presence, while the low $\alpha=0.0006$ value yields a UAP consumption rate of the same magnitude as the production rate. In addition, the long-time increase of heterotrophs for $\alpha=0.01$ and $\alpha=0.03$ seen in Figure 6 is explained by a slowly increasing UAP degradation rate (more negative rate value), which is a result not of changing autotrophic UAP production (which is not dramatically different from the other cases), but a result of the less-extreme physiological behavior of the heterotrophs. We see that extreme parameter values quickly move the heterotroph species into a regime of survival or extinction, whereas more moderate parameter values yield dynamic behavior on a longer time scale.

\subsection{Comparison of Parameter Sets}

We can conclude from the results of the previous sections that multiple parameter sets are able to yield the roughly 1:1 heterotroph-to-autotroph fraction reported by Kindaichi et al. (2004). Therefore, we further explored the behavior of eight example parameter sets, which are given in Table V. We chose three parameter sets from the Laspidou and Rittmann (2002a) (LR) $\hat{q}$ and one from the COD- $\hat{q}$ studies, and four parameter sets with $\hat{q}$ values lying between these extremes. Figure 8(a) shows the evolution in time of the relative species fraction for the eight sets of interest. From this plot, one sees that most of the 
parameter sets yield a slightly higher heterotroph than autotroph presence in the biofilm, which is due to the balance of growth and decay rates for the two species (Table III). Another important trend is that the time course of the relative fractions of the LR-1, MID-2, MID-4, and COD parameter sets tend toward the same value. Also of note are the families of LR and MID curves: the subsets of these two cases, as seen in Table $\mathrm{V}$, show only a variation in $K_{\mathrm{UAP}}$ and $K_{\mathrm{BAP}}$ values, with $\hat{q}_{\mathrm{UAP}}$ and $\hat{q}_{\mathrm{BAP}}$ kept constant. The time course of the relative fraction for these sets fits with the conclusions of Sections 3.3 and 3.4: LR-1 and MID-1 have low $K$ values and show a high $X_{h} / X_{a}$ fraction, LR-3 and MID-3 have higher $K$ values and show the lowest fraction, and LR-2 and MID-2 lie in between.

Plotted in Figure $8(\mathrm{~b})$ are the points from Table $\mathrm{V}$ in a $K_{\mathrm{UAP}}-\hat{q}_{\mathrm{UAP}}$ space with regions labeled based on the relative $X_{h} / X_{a}$ fraction after 90 days of evolution. One may easily see that most of the parameter sets lie nearly on a line that divides the parameter space in two: regions where the heterotrophs have a higher presence than autotrophs (due to a high growth rate) and regions where the heterotrophs have a lower presence. Parameter sets yielding $r$-strategist heterotrophs (higher values of $\hat{q}_{\mathrm{UAP}}$, such as the COD- $\hat{q}$ parameters in Section 3.4) lie toward the right edge of Figure 8(b), showing that the value of $K$ is less important in determining heterotroph subsistence when growth rates are high. $K$-strategist parameter sets (such as those for the $\hat{q}$ variations in Section 3.2) lie toward the bottom of the plot, so that for low 
values of $K_{\mathrm{UAP}}$ the particular value of $\hat{q}$ has little impact on the success of the heterotroph population. In contrast, the species balance for parameter sets such as the LR- $\hat{q}$ in Section 3.3 shows a strong dependence on the value of $K_{\mathrm{UAP}}$, and high $K$ values (such as set LR-3) may prove extremely prohibitive to heterotroph subsistence on SMP; this was also seen in Sections 3.3 and 3.4 and in Figure 5.

The dashed line plotted in Figure 8(b) separates the two regions of species coexistence and heterotroph failure. By assuming that, for roughly 1:1 heterotrophto-autotroph ratio, the net specific growth rates of each species will be equivalent, we may derive the slope of this line as follows. For simplicity, we consider the balance between utilization of $S_{a}$ by the autotrophs and the utilization of UAP by the heterotrophs, and using the corresponding growth terms from Table I we have the following:

$$
\begin{aligned}
& \hat{q}_{S_{a}} Y_{S_{a}}\left(\frac{S_{a}}{K_{S_{a}}+S_{a}}\right)\left(\frac{A}{K_{a}+A}\right) \rho_{a} X_{a} \approx \\
& \hat{q}_{\mathrm{UAP}} Y_{p}\left(\frac{\mathrm{UAP}}{K_{\mathrm{UAP}}+\mathrm{UAP}}\right)\left(\frac{A}{K_{h}+A}\right) \rho_{h} X_{h} .
\end{aligned}
$$

We rearrange the above to obtain:

$$
K_{\mathrm{UAP}}=\frac{Y_{p}}{\hat{q}_{S_{a}} Y_{S_{a}}} \cdot \frac{\mathrm{UAP}}{\left(\frac{S_{a}}{K_{S_{a}}+S_{a}}\right)} \cdot \frac{\left(\frac{A}{K_{h}+A}\right)}{\left(\frac{A}{K_{a}+A}\right)} \cdot \frac{\rho_{h}}{\rho_{a}} \cdot \frac{X_{h}}{X_{a}} \cdot \hat{q}_{\mathrm{UAP}}-\mathrm{UAP} .
$$

Now this relation depends on concentrations that vary in space, but because we are interested in the global relation between parameters we integrate over 
the entire biofilm region $\Omega_{B}$ :

$$
\begin{aligned}
\int_{\Omega_{B}} K_{\mathrm{UAP}} d \Omega= & \int_{\Omega_{B}}\left[\frac{Y_{p}}{\hat{q}_{S_{a}} Y_{S_{a}}} \cdot \frac{\rho_{h}}{\rho_{a}} \cdot \frac{\mathrm{UAP}}{\left(\frac{S_{a}}{K_{S_{a}}+S_{a}}\right)} \cdot \frac{\left(\frac{A}{K_{h}+A}\right)}{\left(\frac{A}{K_{a}+A}\right)} \cdot \frac{X_{h}}{X_{a}} \cdot \hat{q}_{\mathrm{UAP}}\right] d A \\
& -\int_{\Omega_{B}} \mathrm{UAP} d \Omega .
\end{aligned}
$$

Finally, if we define the biofilm area to be $A_{\Omega}$ and take constants out of the integrals, we may write a linear relation between $K_{\mathrm{UAP}}$ and $\hat{q}_{\mathrm{UAP}}$ that will help make sense of Figure 8(b):

$$
\begin{aligned}
K_{\mathrm{UAP}}= & {\left[\frac{1}{A_{\Omega}} \cdot \frac{Y_{p}}{\hat{q}_{S_{a}} Y_{S_{a}}} \cdot \frac{\rho_{h}}{\rho_{a}} \cdot \int_{\Omega_{B}} \frac{\mathrm{UAP}}{\left(\frac{S_{a}}{K_{S_{a}}+S_{a}}\right)} \cdot \frac{\left(\frac{A}{K_{h}+A}\right)}{\left(\frac{A}{K_{a}+A}\right)} \cdot \frac{X_{h}}{X_{a}} d A\right] \cdot \hat{q}_{\mathrm{UAP}} } \\
& -\frac{1}{A_{\Omega}} \int_{\Omega_{B}} \mathrm{UAP} d \Omega .
\end{aligned}
$$

From this relation, it is apparent that the $K_{\mathrm{UAP}}$ value for a given heterotrophto-autotroph ratio should depend linearly on $\hat{q}_{\mathrm{UAP}}$.

Note that the relation in (8) is made even simpler for this particular system, for the fraction $\rho_{h} / \rho_{a}$ is unity (Table II), as is the fraction of Monod terms for the electron acceptor $(A)$ due to the equality between $K_{h}$ and $K_{a}$ (Table III). In addition, we found for this system that the following term was of order one for all cases but LR-3 in Table V (data not shown):

$$
\frac{1}{A_{\Omega}} \int_{\Omega_{B}} \frac{\mathrm{UAP}}{\left(\frac{S_{a}}{K_{S_{a}}+S_{a}}\right)} \cdot \frac{X_{h}}{X_{a}} d A \approx 1 .
$$


(LR-3 does not fit this relation because in this case the $X_{h} / X_{a}$ ratio is much less than 1, leading to the above integral being quite small.) Therefore for most cases we may use (9) to simplify the relation in (8) to the following:

$$
K_{\mathrm{UAP}}=\left[\frac{Y_{p}}{\hat{q}_{S_{a}} Y_{S_{a}}}\right] \cdot \hat{q}_{\mathrm{UAP}}-\left[\frac{1}{A_{\Omega}} \int_{\Omega_{B}} \mathrm{UAP} d \Omega\right]
$$

The dashed line in Figure $8(\mathrm{~b})$ is plotted using the slope $Y_{p} / \hat{q}_{S_{a}} Y_{S_{a}}$ that appears in (10), and the line reinforces the important trend that $K_{\mathrm{UAP}}$ and $\hat{q}_{\mathrm{UAP}}$ are related in yielding a given heterotroph-to-autotroph ratio. Recall that the LR-1, MID-2, MID-4, and COD parameter sets seemed to follow a similar trend in Figure 8(a); these parameter sets lie parallel to the 1:1 dashed line in Figure 8(b). In addition, Figure 8(a) shows that these parameter sets all yield a slight heterotroph advantage, and therefore the four points lie just below the dashed line of coexistence; this is partly due to the fact that for our system the heterotrophic decay rate is lower than for autotrophs (Table III). Point LR-2 seems to lie on the incorrect side of the dashed line based on its 90-day value in Figure 8(a), but we note that the LR-2 ratio shows an upward trend and no evidence of the plateau seen in Figure 4 or the other cases in Figure 8(a); if this case were run for a longer time period these results suggest that the long-time trend would follow LR-1. Overall, these results reinforce that the relation in (10) describes the species balance quite well.

In addition to using (10) to separate the different regions in phase space, we 
also used this equation to evaluate the "predicted" value of $K_{\mathrm{UAP}}$ based on the utilization rate $\hat{q}_{\mathrm{UAP}}$ and the resulting heterotroph-to-autotroph ratio for each case in Table V. In Table VI, the "predicted" value is listed along with the actual value used. We find that knowing the final heterotroph-to-autotroph ratio along with an estimate of the UAP/Monod term in (9) might allow one to make a reasonable estimate of the value of $K_{\mathrm{UAP}}$ that made this particular ratio possible. However, this is only possible for those parameter sets that yield an $X_{h} / X_{a}$ ratio of the order 1 , because the simplifying assumption in (9) falls apart if the ratio differs from 1.

Finally, this parameter-set comparison shows that the plateau in the heterotrophto-autotroph ratio that was first mentioned in Section 3.2 (and which is seen most prevalently in Figure 4) is indeed a result of the balance in SMP production by the autotrophs and SMP consumption by the heterotrophs. A particular set of growth parameters will lead to a particular plateau value, and though no closed-form expression is possible to predict the final plateau ratio, Figure 8(b) provides a means of predicting the final species balance of the system.

In general, this comparison of parameter sets has shown that variations in SMP parameters will affect the ability of heterotrophs to subsist on low concentrations of UAP and BAP, and will also affect the time required before the two species reach a stable coexistence. 


\subsection{Split Inoculations}

While it is apparent that the range of values explored in the previous sections show that both $r$ - and $K$-strategies allow heterotrophs to thrive off autotrophically produced SMP, these explorations were all conducted for a mixed inoculation that nurtures inter-species cooperation. It is not immediately apparent that the ability of heterotrophs to subsist in mixed inoculations translates to separated inoculations, even with the tendency of biofilm colonies to spread laterally (Figure 9). To test the hypothesis that a heterotroph population will subsist in the long term when the initial colony is sufficiently close to an autotroph colony, we explored the evolution of colonies separated by different distances. We inoculated two half-circle colonies of radius $31 \mu \mathrm{m}$, one colony composed of $2 / 3$ autotrophs and the other composed of $2 / 3$ heterotrophs, with both colonies comprised of $1 / 3$ inert biomass to yield an overall mass distribution of $1 / 3$ to each species as in Table II. We then explored variations in the initial separation of these two colonies.

Figure 9 shows the evolution of one inoculation run (the LR-1 case in Table $\mathrm{V}, K$-strategist parameters), with the autotroph and heterotroph colonies initially separated by a distance of $150 \mu m, 5$ times the initial colony radius. Shown in the figure are the contours for the concentration of UAP in the domain; also shown is the biofilm interface location. The UAP concentration plotted in Figure 9 captures the behavior of the two active bacterial species, 
with the peaks (that first increase and then decrease in time) capturing autotroph growth and the troughs capturing heterotroph growth (a result of UAP consumption).

After two days of evolution, the heterotroph colony has decreased slightly in height, because the concentration of UAP has only recently become nonzero within the heterotroph colony. As the autotroph colony grows so that the heterotroph colony is fully within the distance of the boundary layer (which has thickness $L_{l s}$ ), the heterotrophs are provided with ample SMP. The biofilm grows to a flat state by 10 days, and the flat profile is a result of the initial dominance by the slower-growing autotrophs (Picioreanu et al., 1998), as well as the heterotrophs receiving electron donor from within the biofilm. After 10 days, though the shape of the biofilm-liquid interface no longer varies, the biofilm continues to change composition internally, as seen by the varying UAP concentration field.

We also considered the impact of split inoculation on growth governed by different parameter values. We used a subset of the values as given in Table $\mathrm{V}$, and inoculated with two colonies (one with autotrophs and one with heterotrophs, as above) of radius $31 \mu m$ with their centers $150 \mu m$ apart. Figure 10 plots the time evolution of the heterotroph-to-autotroph ratio, and a comparison with Figure 8(a) shows the impact that the split inoculation has on the survival of the heterotroph population. The heterotroph population drops quickly in comparison to autotroph presence, similar to the mixed case, and in all cases 
drops as much or more than the mixed case. Rather non-intuitively, though, recovery from this drop occurs more quickly in the split inoculation case, and the long-term trend is a higher heterotroph-to-autotroph ratio than is found in the mixed inoculation tests. Because the only difference in the runs is the geometry of the initial species distribution (separated rather than mixed), we must conclude that the split inoculation allows for a more thriving heterotroph population because the competition for space (as well as the common electron acceptor) is more limited than in the mixed case: the heterotrophs are able to expand more easily when the autotrophs occupy a different spatial region. The initial separation is not enough to limit heterotroph growth because diffusion is so effective in delivering SMP to all portions of the biofilm; the only case in which heterotrophs will have trouble surviving is in cases of more distant inoculation, where the heterotroph colony is lost before the autotroph population is close enough to provide SMP for heterotrophic growth.

To study the effect of inoculation distance in more detail we considered a variation in the inoculation distance for the LR-1 parameter set from Table V. Shown in Figure 11 is the effect of different inoculation distances on the resulting heterotroph-to-autotroph fraction as a function of time. The fraction drops quite quickly for all initial distances, but, as was seen in Figure 10, each case eventually recovers. In this case, though, the recovery rate is proportional to the inoculation distance. One sees in Figure 11 that the spacing of different inoculation distances is fairly regular, suggesting that even larger initial 
separations may prove surmountable and allow heterotrophic subsistence. We may conclude that so long as a heterotroph colony is provided some source of organic nutrients, whether COD from the bulk or SMP from autotrophic growth, the heterotroph colony has a strong potential to survive over long times.

\section{Conclusions}

In this study, we explored how variations in SMP parameters affect the growth of a biofilm and the resulting autotroph and heterotroph distributions, and we also explored the ability of $r$ - and $K$-strategist heterotroph species to subsist solely on autotrophically produced SMP. In order to carry out this study, we constructed a new multi-species, multi-component biofilm model that makes possible a study of the interaction between autotrophic and heterotrophic species via SMP and EPS; previously published models of autotroph-heterotroph systems are unable to capture these dynamics.

We found that the previously published SMP parameters given by Noguera et al. (1994) and Laspidou and Rittmann (2002a) are not appropriate to model the findings of Kindaichi et al. (2004), but, using the new model to explore the space of $K$ and $\hat{q}$ variations, we show that a range of parameters is able to describe what is seen experimentally. This range suggests that these multispecies biofilms may consist of heterotrophs that fall into either or both of the 
$r$-strategist or $K$-strategist groups, though for the low SMP utilization rates given in Laspidou and Rittmann (2002a) it is likely the the heterotrophs tend to be $K$-strategists. In addition, we found that heterotrophic bacteria that grow through the utilization of SMP from autotrophs can survive even when their initial position is away from the autotrophs, although greater distance slows the growth of the heterotroph population.

\section{Acknowledgments}

BVM and DLC acknowledge the support of NSF grants DMS-0610546, and DMS-0636574. 


\section{References}

Alpkvist, E. and Klapper, I. (2007a). Description of mechanical response including detachment using a novel particle model of bifoilm/flow interaction. Wat. Sci. Tech., In Press.

Alpkvist, E. and Klapper, I. (2007b). A multidimensional multispecies continuum model for heterogeneous biofilm development. Bull. Math. Bio., 69:765-789.

Alpkvist, E., Picioreanu, C., van Loosdrecht, M. C. M., and Heyden, A. (2006). Three-dimensional biofilm model with individual cells and continuum EPS matrix. Biotech. Bioeng., 94(5):961-979.

Andrews, J. H. and Harris, R. F. (1986). r and k-selection and microbial ecology. Adv. Microb. Ecol., 9:99-147.

Barker, D. J. and Stuckey, D. C. (1999). A review of soluble microbial products (SMP) in wastewater treatment systems. Wat. Res., 33(14):3063-3082.

Bishop, P. L., Zhang, T. C., and Fu, Y.-C. (1995). Effects of biofilm structure, microbial distributions, and mass transport on biodegradation processes. Wat. Sci. Tech., 31(1):143-152.

Characklis, W. G. and Marshall, K. C. (1990). Biofilms. John Wiley \& Sons, Inc., New York.

Dockery, J. and Klapper, I. (2001). Finger formation in biofilm layers. SIAM J. Appl. Math., 62(3):853-869.

Duddu, R., Bordas, S., Chopp, D. L., and Moran, B. (2007). A combined 
extended finite element and level set method for biofilm growth. Int. J. Num. Meth. Eng., 74(5):848-870.

Duddu, R., Chopp, D. L., and Moran, B. (2008). A two-dimensional continuum model of biofilm growth incorporating fluid flow and shear stress based detachment. Biotech. Bioeng., To Appear.

Eberl, H., Morgenroth, E., Noguera, D., Picioreanu, C., Rittmann, B. E., van Loosdrecht, M. C. M., and Wanner, O. (2006). Mathematical modeling of biofilms. Technical Report 18, Intl. Wat. Assoc., London, UK.

Furumai, H. and Rittmann, B. E. (1992). Advanced modeling of mixed populations of heterotrophs and nitrifiers considering the formation and exchange of soluble microbial products. Wat. Sci. Tech., 26:493-493.

Furumai, H. and Rittmann, B. E. (1994). Interpretation of bacterial activities in nitrification filters by a biofilm model considering the kinetics of soluble microbial products. Wat. Sci. Tech., 30(11):147-156.

Horn, H., Reiff, H., and Morgenroth, E. (2003). Simulation of growth and detachment in biofilm systems under defined hydrodynamic conditions. Biotech. Bioeng., 81(5):607-617.

Kindaichi, T., Ito, T., and Okabe, S. (2004). Ecophysiological interaction between nitrifying bacteria and heterotrophic bacteria in autotrophic nitrifying biofilms as determined by microautoradiography-fluorescence in situ hybridization. Appl Environ Microbiol, 70(3):1641-1650.

Kissel, J. C., McCarty, P. L., and Street, R. L. (1984). Numerical simulation of mixed-culture biofilm. J. Env. Eng., 110(2):393-411. 
Klapper, I., Rupp, C. J., Cargo, R., Purvedorj, B., and Stoodley, P. (2002). Viscoelastic fluid description of bacterial biofilm material properties. Biotech. Bioeng., 80(3):289-296.

Kreft, J.-U. (2004). Biofilms promote altruism. Microbiology, 150:2751-2760.

Kreft, J.-U. and Bonhoeffer, S. (2005). The evolution of groups of cooperating bacteria and the growth rate versus yield trade-off. Microbiology, 151(3):637-641.

Kreft, J.-U. and Wimpenny, J. W. T. (2001). Effect of EPS on biofilm structure and function as revealed by an individual-based model of biofilm growth. Wat. Sci. Tech., 43(6):135-141.

Laspidou, C. S. and Rittmann, B. E. (2002a). Non-steady state modeling of extracellular polymeric substances, soluble microbial products, and active and inert biomass. Wat. Res., 36:1983-1992.

Laspidou, C. S. and Rittmann, B. E. (2002b). A unified theory for extracellular polymeric substances, soluble microbial products, and active and inert biomass. Wat. Res., 36:2711-2720.

Laspidou, C. S. and Rittmann, B. E. (2004a). Evaluating trends in biofilm density using the UMCCA model. Wat. Res., 38:3362-3372.

Laspidou, C. S. and Rittmann, B. E. (2004b). Modeling the development of biofilm density including active bacteria, inert biomass, and extracellular polymeric substances. Wat. Res., 38:3349-3361.

Leveque, R. J. and Li, Z. (1994). The immersed interface method for elliptic equations with discontinuous coefficients and singular sources. SIAM J. 
Num. Anal., 31(4):1019-1044.

Morgenroth, E. and Wilderer, P. A. (2000). Influence of detachment mechanisms on competition in biofilms. Wat. Res., 34(2):417-426.

Noguera, D., Araki, N., and Rittmann, B. E. (1994). Soluble microbial products (SMP) in anaerobic chemostats. Biotech. Bioeng., 44:1040-1047.

Okabe, S., Hiratia, K., Ozawa, Y., and Watanabe, Y. (1996). Spatial microbial distributions of nitrifiers and heterotrophs in mixed-population biofilms. Biotech. Bioeng., 50:24-35.

Osher, S. and Sethian, J. A. (1988). Fronts propagating with curvaturedependent speed: Algorithms based on hamilton-jacobi formulations. $J$. Comput. Phys, 79:12-49.

Picioreanu, C., van Loosdrecht, M. C. M., and Heijnen, J. J. (1998). Mathematical modeling of biofilm structure with a hybrid differential-discrete cellular automata approach. Biotech. Bioeng., 58(1):101-116.

Picioreanu, C., van Loosdrecht, M. C. M., and Heijnen, J. J. (1999). Discretedifferential modelling of biofilm structure. Wat. Sci. Tech., 39(7):115-122.

Rauch, W., Vanhooren, H., and Vanrolleghem, P. A. (1999). A simplified mixed-culture biofilm model. Wat. Res., 33(9):2148-2162.

Rittmann, B. E. (2004). Biofilms in the water industry. In Ghannoum, M. A. and O’Toole, G. A., editors, Microbial Biofilms, pages 359-378. ASM Press, Washington, D.C.

Rittmann, B. E. and Manem, J. A. (1992). Development and experimental evaluation of a steady-state, multispecies biofilm model. Biotech. Bioeng., 
39:914-922.

Rittmann, B. E. and McCarty, P. L. (2001). Environmental Biotechnology: Principles and Applications. McGraw-Hill, New York.

Rittmann, B. E., Stilwell, D., and Ohashi, A. (2002). The transient-state, multiple-species biofilm model for biofiltration processes. Wat. Res., $36: 2342-2356$.

Sethian, J. A. (1999). Level Set Methods and Fast Marching Methods. Cambridge Monographs on Applied and Computational Mathematics. Cambridge University Press, New York.

Stoodley, P., Cargo, R., Rupp, C. J., Wilson, S., and Klapper, I. (2002a). Biofilm material properties as related to shear-induced deformation and detachment phenomena. J. Ind. Microbio. Biotech., 29(6):361-367.

Stoodley, P., Sauer, K., Davies, D. G., and Costerton, J. W. (2002b). Biofilms as complex differentiated communities. Ann. Rev. Microbio., 56:187-209.

Velicer, G. J., Schmidt, T. M., and Lenski, R. E. (1999). Application of traditional and phylogenetically based comparative methods to test for a trade-off in bacterial growth rate at low versus high substrate concentration. Microb Ecol, 38(3):191-200.

Wanner, O. and Gujer, W. (1986). A multispecies biofilm model. Biotech. Bioeng., 28:314-328.

Wanner, O. and Reichert, P. (1995). Mathematical modeling of mixed-culture biofilms. Biotech. Bioeng., 49:172-184.

Wood, B. D. and Whitaker, S. (2000). Cellular growth in biofilms. Biotech. 
Bioeng., 64(6):656-670.

Xavier, J. and Foster, K. R. (2007). Cooperation and conflict in microbial biofilms. Proc. Natl. Acad. Sci., 104(3):876-881.

Xavier, J., Picioreanu, C., and van Loosdrecht, M. C. M. (2005). A general description of detachment for multidimensional modelling of biofilms. Biotech. Bioeng., 91(6):651-669.

Zhang, T. C., Fu, Y.-C., and Bishop, P. L. (1994). Competition in biofilms. Wat. Sci. Tech., 29(10-11):263-270. 


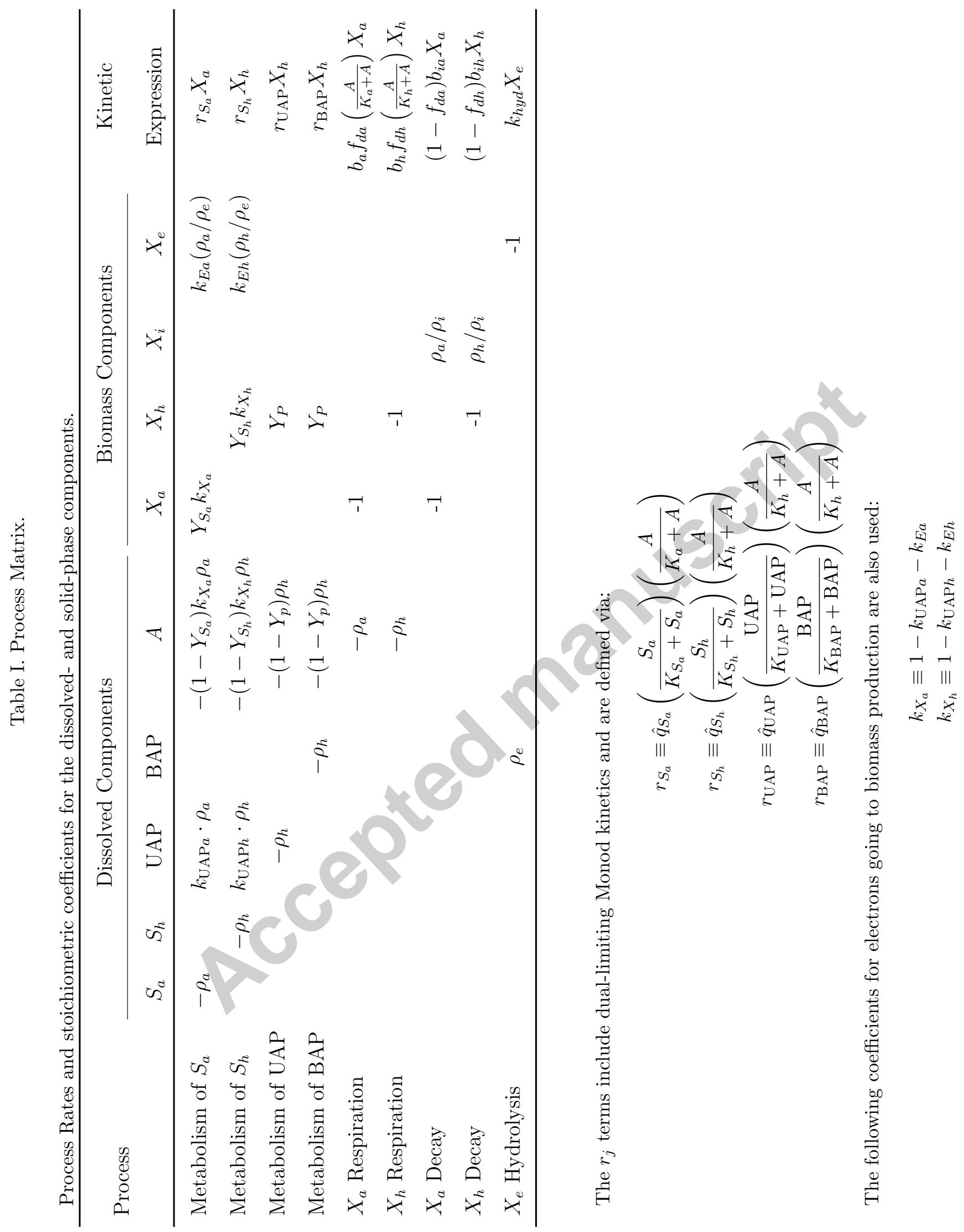


Table II

Physical and system parameters.

\begin{tabular}{|c|c|c|c|c|}
\hline Name & Description & Value & Units & Source \\
\hline$S_{a L}$ & $S_{a}($ Ammonium) concentration in bulk fluid flow & 59.4 & $m g \mathrm{COD} / L$ & 2 \\
\hline$S_{h L}$ & $S_{h}$ (Organics) concentration in bulk fluid flow & 0 & $m g \mathrm{COD} / L$ & A \\
\hline $\mathrm{UAP}_{L}$ & UAP concentration in bulk fluid flow & 0 & $m g \mathrm{COD} / L$ & $\mathrm{~A}$ \\
\hline $\mathrm{BAP}_{L}$ & BAP concentration in bulk fluid flow & 0 & $m g \mathrm{COD} / L$ & A \\
\hline$A_{L}$ & $A$ (Oxygen) concentration in bulk fluid flow & 8 & $m g \mathrm{O}_{2} / L$ & 2 \\
\hline$X_{a 0}$ & Initial volume fraction of autotrophs & 0.33 & - & 2 \\
\hline$X_{h 0}$ & Initial volume fraction of heterotrophs & 0.33 & - & 2 \\
\hline$X_{i 0}$ & Initial volume fraction of inerts & 0.33 & & 2 \\
\hline$X_{e 0}$ & Initial volume fraction of EPS & 0 & & 2 \\
\hline$\rho_{a}$ & Autotroph density & 0.05 & $m g \mathrm{COD} / \mathrm{mm}^{3}$ & 1 \\
\hline$\rho_{h}$ & Heterotroph density & 0.05 & $m g \mathrm{COD} / \mathrm{mm}^{3}$ & 1 \\
\hline$\rho_{i}$ & Inerts density & 0.05 & $m g \mathrm{COD} / m m^{3}$ & 1 \\
\hline$\rho_{e}$ & EPS density & 0.05 & $m g \mathrm{COD} / m^{3}$ & 1 \\
\hline$k_{\text {hyd }}$ & Hydrolysis rate & 0.17 & $d^{-1}$ & 3 \\
\hline$k_{\mathrm{det}}$ & Detachment strength & 2.0 & $(m m \cdot d)^{-1}$ & A \\
\hline$D_{S_{a}}$ & $S_{a}$ diffusion coefficient & 186 & $m m^{2} / d$ & 2 \\
\hline$D_{S_{h}}$ & $S_{h}$ diffusion coefficient & 104 & $m m^{2} / d$ & 2 \\
\hline$D_{\mathrm{UAP}}$ & UAP diffusion coefficient & 138 & $m m^{2} / d$ & 4 \\
\hline$D_{\mathrm{BAP}}$ & BAP diffusion coefficient & 138 & $m m^{2} / d$ & 4 \\
\hline$D_{A}$ & $A$ diffusion coefficient & 219 & $m m^{2} / d$ & 2 \\
\hline$\gamma$ & Ratio of interior/exterior diffusion coefficients & 0.8 & - & 1 \\
\hline$L_{l s}$ & Thickness of boundary layer above biofilm & 0.05 & $m m$ & 1 \\
\hline
\end{tabular}

Sources for values were 1: Rittmann and McCarty (2001), 2: Wanner and Gujer (1986), 3: Laspidou and Rittmann (2002a,b), 4: Laspidou and Rittmann (2004a,b), A: assumed. 
Table III

Reaction parameters.

\begin{tabular}{|c|c|c|c|c|}
\hline Name & Description & Value & Units & Source \\
\hline$\hat{q}_{S_{a}}$ & Max $S_{a}$ utilization rate & 19.73 & $m g \mathrm{COD} / m g \mathrm{COD} / d$ & 2 \\
\hline$Y_{S_{a}}$ & True yield for $S_{a}$ utilization & 0.048 & $m g \mathrm{COD} / m g \mathrm{COD}$ & 2 \\
\hline$K_{S_{a}}$ & Monod coefficient for $S_{a}$ utilization & 4.57 & $m g \mathrm{COD} / L$ & 2 \\
\hline$k_{\mathrm{UAP} a}$ & UAP formation by $X_{a}$ & 0.05 & $m g \mathrm{COD} / m g \mathrm{COD}$ & 1 \\
\hline$k_{E a}$ & EPS formation by $X_{a}$ & 0.075 & $m g \mathrm{COD} / m g \mathrm{COD}$ & $\mathrm{A}^{*}$ \\
\hline$K_{a}$ & Monod coefficient for $A$ utilization $\left(X_{a}\right)$ & 0.1 & $m g \mathrm{O}_{2} / L$ & 2 \\
\hline$f_{d a}$ & Autotroph biodegradable fraction & 0.8 & - & 1 \\
\hline$b_{a}$ & Autotroph endogenous decay & 0.0625 & $d^{-1}$ & 2 \\
\hline$b_{i a}$ & Autotroph inactivation rate & 0.5 & $d^{-1}$ & 2 \\
\hline$\hat{q}_{S_{h}}$ & Max $S_{h}$ utilization rate & 12.1 & $m g \mathrm{COD} / m g \mathrm{COD} / d$ & 2 \\
\hline$Y_{S_{h}}$ & True yield for $S_{h}$ utilization & 0.4 & $m g \mathrm{COD} / m g \mathrm{COD}$ & 2 \\
\hline$K_{S_{h}}$ & Monod coefficient for $S_{h}$ utilization & 5 & $m g \mathrm{COD} / L$ & 2 \\
\hline$k_{\mathrm{UAP} h}$ & UAP formation by $X_{h}$ & 0.12 & $m g \mathrm{COD} / m g \mathrm{COD}$ & 3 \\
\hline$k_{E h}$ & EPS formation by $X_{h}$ & 0.18 & $m g \mathrm{COD} / m g \mathrm{COD}$ & 3 \\
\hline$K_{h}$ & Monod coefficient for $A$ utilization $\left(X_{h}\right)$ & 0.1 & $m g O_{2} / L$ & 2 \\
\hline$f_{d h}$ & Heterotroph biodegradable fraction & 0.8 & - & 1 \\
\hline$b_{h}$ & Heterotroph endogenous decay & 0.025 & $d^{-1}$ & 1 \\
\hline$b_{i h}$ & Heterotroph inactivation rate & 0.05 & $d^{-1}$ & 1 \\
\hline$\hat{q}_{\mathrm{UAP}}$ & Max UAP utilization rate & 1.27 & $m g \mathrm{COD} / m g \mathrm{COD} / d$ & $3^{\dagger}$ \\
\hline$\hat{q}_{\mathrm{BAP}}$ & Max BAP utilization rate & 0.07 & $m g \mathrm{COD} / m g \mathrm{COD} / d$ & $3^{\dagger}$ \\
\hline$Y_{p}$ & True yield for SMP utilization & 0.45 & $m g \mathrm{COD} / m g \mathrm{COD}$ & 3 \\
\hline$K_{\mathrm{UAP}}$ & Monod coefficient for UAP utilization & 100 & $m g \mathrm{COD} / L$ & $3^{\dagger}$ \\
\hline$K_{\mathrm{BAP}}$ & Monod coefficient for BAP utilization & 85 & $m g \mathrm{COD} / L$ & $3^{\dagger}$ \\
\hline$\alpha$ & Coefficient on $K$ for parameter variations & Variable & - & $\mathrm{A}$ \\
\hline
\end{tabular}

Sources for values were 1: Rittmann and McCarty (2001), 2: Wanner and Gujer (1986), 3: Laspidou and Rittmann (2002a,b), 4: Laspidou and Rittmann (2004a,b), A: assumed. ${ }^{*} k_{E a}$ was calculated assuming the same $k_{E} / k_{\mathrm{UAP}}$ ratio for the autotrophs as for the heterotrophs. ${ }^{\dagger}$ These parameters were varied in the studies here, but the $\hat{q}_{\mathrm{UAP}} / \hat{q}_{\mathrm{BAP}}$ and $K_{\mathrm{UAP}} / K_{\mathrm{BAP}}$ ratios were kept constant. 
Table IV

Values used in the $\hat{q}$-variation study

\begin{tabular}{ccc}
\hline Case & $\hat{q}_{\mathrm{UAP}}$ & $\hat{q}_{\mathrm{BAP}}$ \\
\hline 1 & 1.27 & 0.07 \\
2 & 4 & 0.22 \\
3 & 6 & 0.33 \\
4 & 8 & 0.44 \\
5 & 10 & 0.55 \\
6 & 12.1 & 0.67 \\
\hline
\end{tabular}

Units for both $\hat{q}_{\mathrm{UAP}}$ and $\hat{q}_{\mathrm{BAP}}$ are: $[m g \mathrm{COD} / m g \mathrm{COD} / d]$.

Table V

Parameter values plotted in Figure 8

\begin{tabular}{ccccc}
\hline Label & $\hat{q}_{\mathrm{UAP}}$ & $K_{\mathrm{UAP}}$ & $\hat{q}_{\mathrm{BAP}}$ & $K_{\mathrm{BAP}}$ \\
\hline Units & {$[m g \mathrm{COD} / m g \mathrm{COD} / d]$} & {$[m g \mathrm{COD} / L]$} & {$[m g \mathrm{COD} / m g \mathrm{COD} / d]$} & {$[m g \mathrm{COD} / L]$} \\
\hline LR-1 & 1.27 & 0.3 & 0.07 & 0.255 \\
LR-2 & 1.27 & 1 & 0.07 & 0.85 \\
LR-3 & 1.27 & 10 & 0.07 & 8.5 \\
MID-1 & 4 & 1 & 0.22 & 0.85 \\
MID-2 & 4 & 2 & 0.22 & 1.7 \\
MID-3 & 4 & 3 & 0.22 & 2.55 \\
MID-4 & 8 & 4 & 0.44 & 3.4 \\
COD & 12.1 & 6 & 0.67 & 5.1 \\
\hline
\end{tabular}

These values are shown in the $K_{\mathrm{UAP}}-\hat{q}_{\mathrm{UAP}}$ space plot in Figure $8(\mathrm{~b})$. 
Table VI

Balance of parameter values for sets in Table V

\begin{tabular}{cccc}
\hline Label & $\hat{q}_{\mathrm{UAP}}$ & Predicted $K_{\mathrm{UAP}}$ & Actual $K_{\mathrm{UAP}}$ \\
\hline Units & {$[m g$ COD $/ m g$ COD $/ d]$} & {$[m g$ COD $/ L]$} & {$[m g$ COD $/ L]$} \\
\hline LR-1 & 1.27 & 0.348 & 0.3 \\
LR-2 & 1.27 & 0.847 & 1 \\
LR-3 & 1.27 & -0.347 & 10 \\
MID-1 & 4 & 1.09 & 1 \\
MID-2 & 4 & 2.18 & 2 \\
MID-3 & 4 & 3.30 & 3 \\
MID-4 & 8 & 4.33 & 4 \\
COD & 12.1 & 6.48 & 6 \\
\hline
\end{tabular}

The relation between $K_{\mathrm{UAP}}$ and $\hat{q}_{\mathrm{UAP}}$ in (8) is used to calculate the "predicted" value of $K_{\mathrm{UAP}}$ based on the utilization rate $\hat{q}_{\mathrm{UAP}}$ and the resulting heterotroph-to-autotroph ratio for each case in Table V. This "predicted" value is listed along with the actual value used in order to show that for cases where the heterotrophs survive in the biofilm, (8) provides a reasonable estimate of the $K_{\mathrm{UAP}}$ value. The LR-3 case yields a poor prediction of $K_{\mathrm{UAP}}$ because the assumption of (9) does not hold, while for the other cases (9) is a more appropriate assumption about the system. This relation underscores that for most parameter sets the balance between production and consumption of UAP is a good measure of the interspecies competition occurring in the biofilm. 


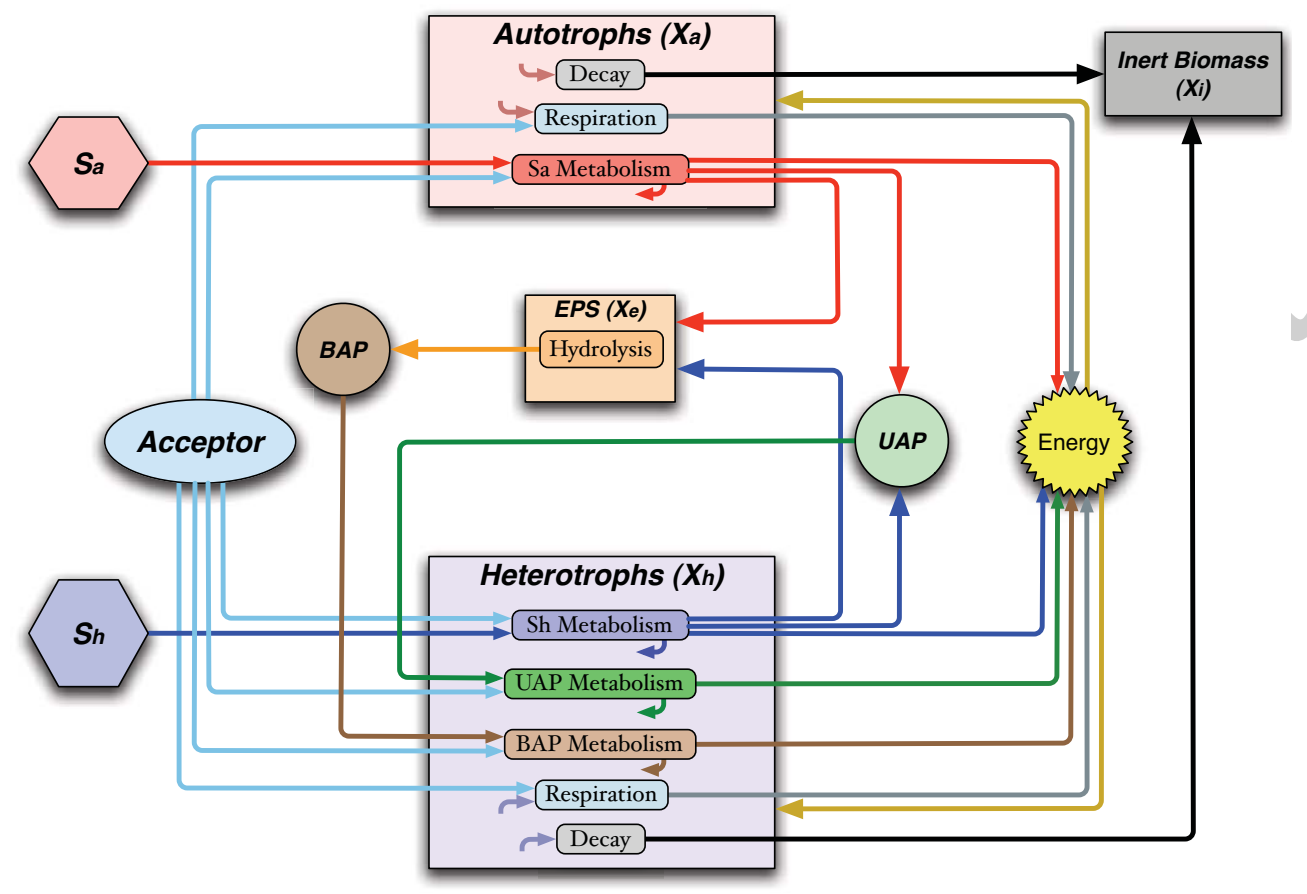

Fig. 1. Model Schematic.

This schematic follows Laspidou and Rittmann (2002a) and describes the interaction of the dissolved components (hexagons, ovals, circles) and solid components (rectangles) in a biofilm. Within the solid components are shown the reactions either carried out by or occurring to the component, with reactants entering and products leaving. Electron donors $S_{a}$ and $S_{h}$ and the electron acceptor $A$ are supplied externally, while an initial bacterial population will yield the remaining components. Energy is not tracked explicitly, but rather is included in the diagram to show that the conversion processes are not $100 \%$ efficient. 


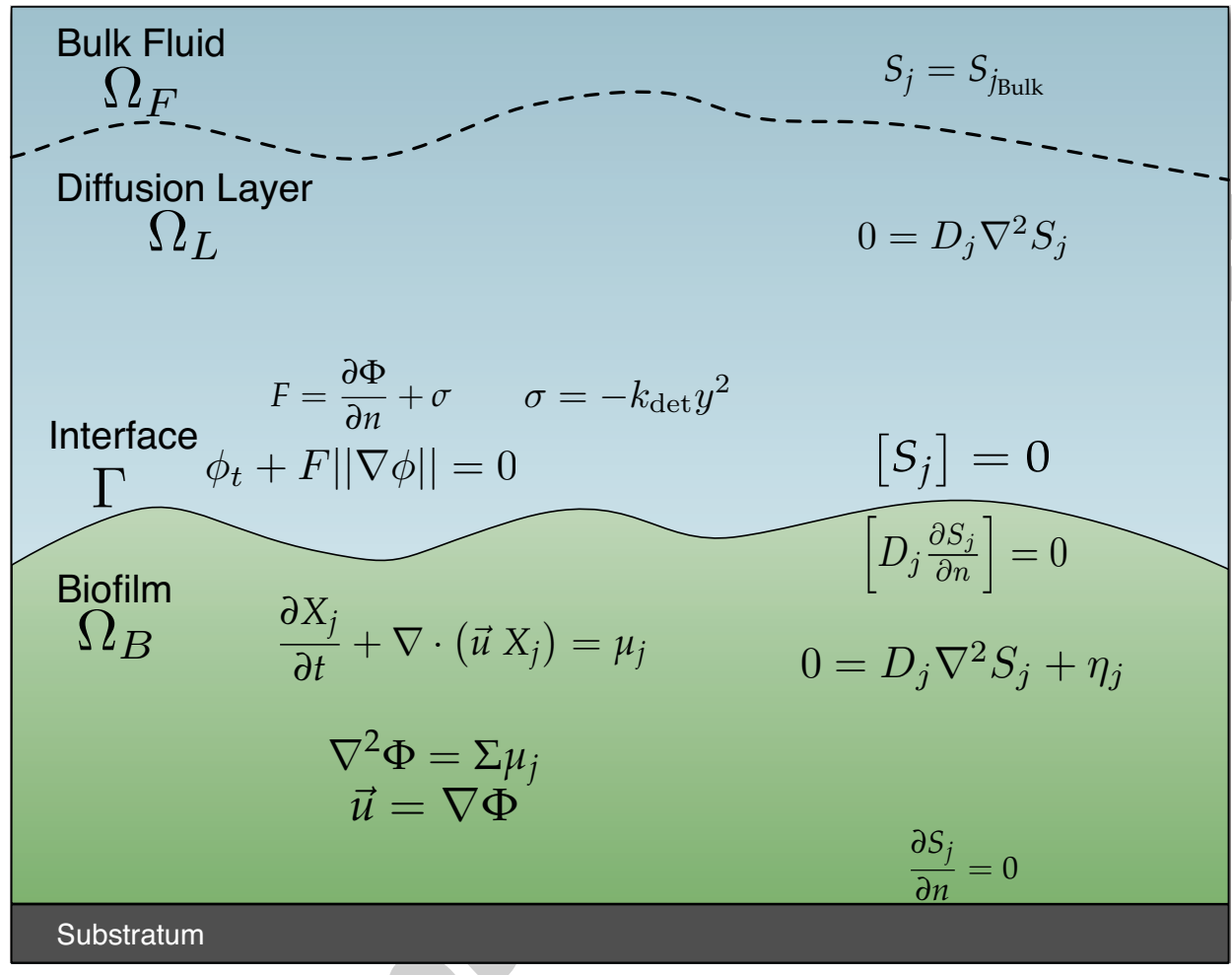

Fig. 2. The compartments in the computational domain.

The solid-component and velocity equations are solved inside the biofilm, and the dissolved-component equations are solved in the biofilm and in the linear diffusion layer. 


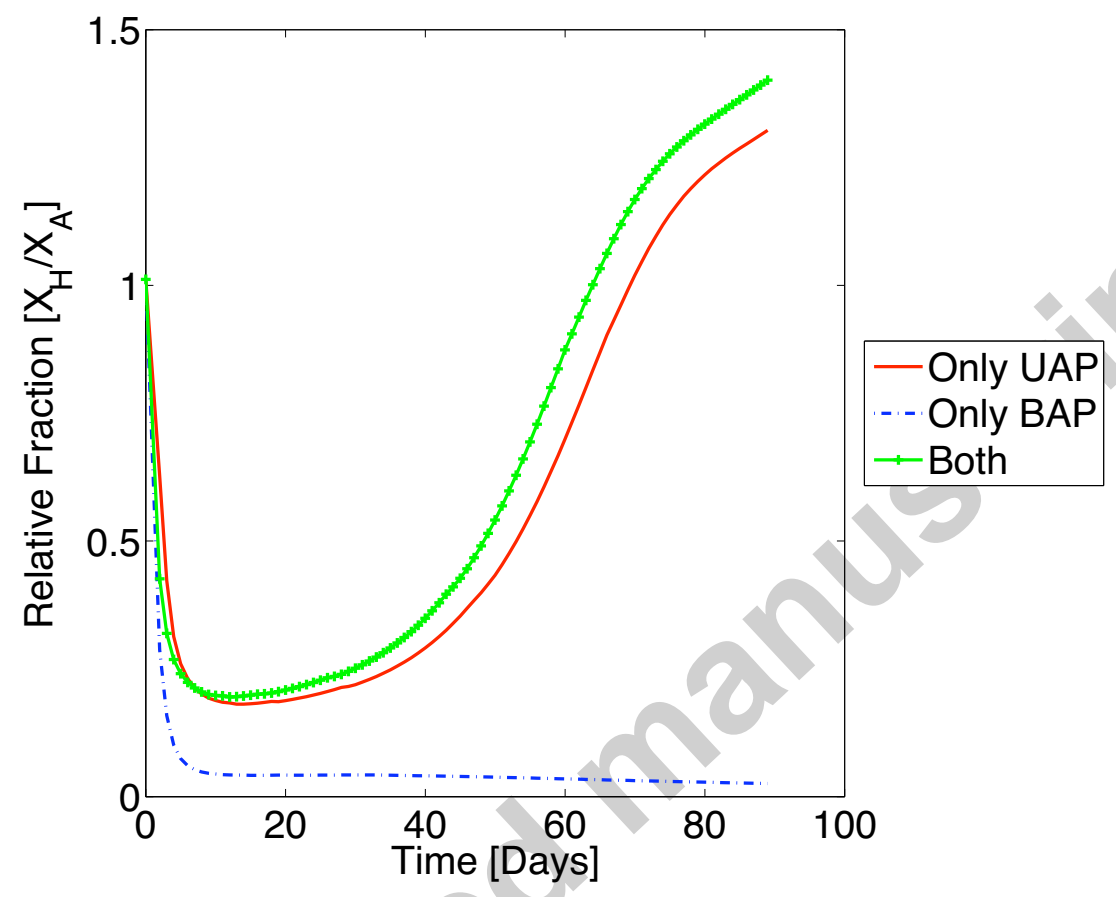

Fig. 3. Effects of heterotrophic growth solely on UAP, BAP, or both.

Plot of relative fraction of heterotrophs to autotrophs as a function of time for growth on UAP alone, BAP alone, and on both SMP types together. For growth only on UAP, the value of $\hat{q}_{\mathrm{BAP}}$ was set to zero, and for growth on BAP alone the value of $\hat{q}_{\mathrm{UAP}}$ was set to zero. It is readily apparent that heterotrophic growth depends almost entirely on utilization of UAP rather than BAP; the only-BAP curve yields almost no heterotrophic survival. 


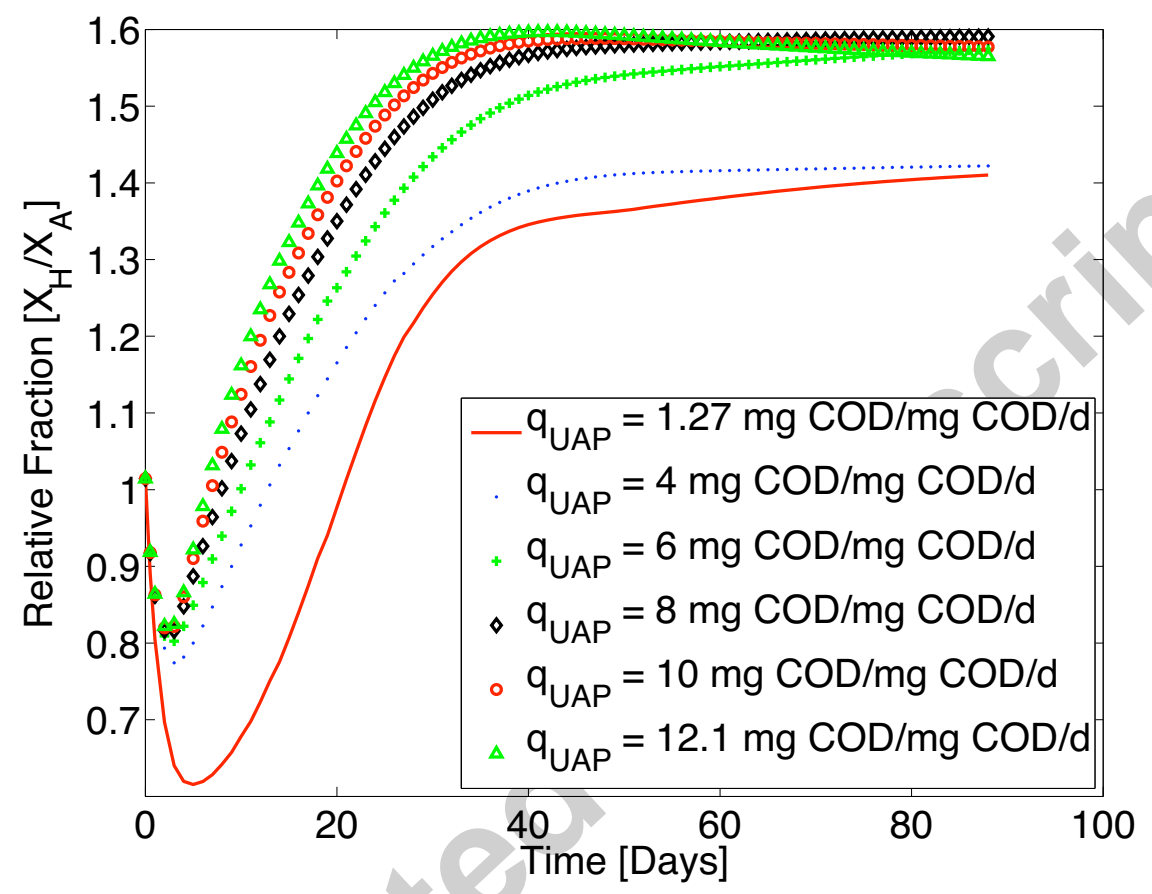

Fig. 4. Variations in $\hat{q}$ for very low $K$.

Plot of relative fraction of heterotrophs to autotrophs as a function of time with varying $\hat{q}$. The values of $\hat{q}_{\mathrm{UAP}}$ and $\hat{q}_{\mathrm{BAP}}$ used are given in Table IV. When $K$ values are not a factor, even low utilization rates yield species coexistence. 
(a) LR-based $\hat{q}_{\mathrm{UAP}}$ and $\hat{q}_{\mathrm{BAP}}$ values

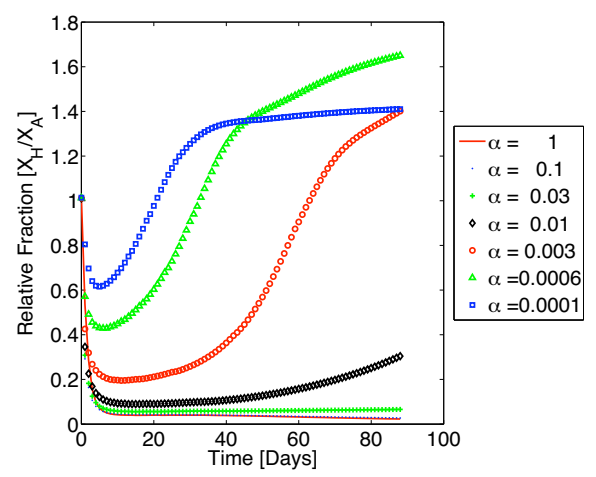

(b) COD-based $\hat{q}_{\mathrm{UAP}}$ with scaled $\hat{q}_{\mathrm{BAP}}$

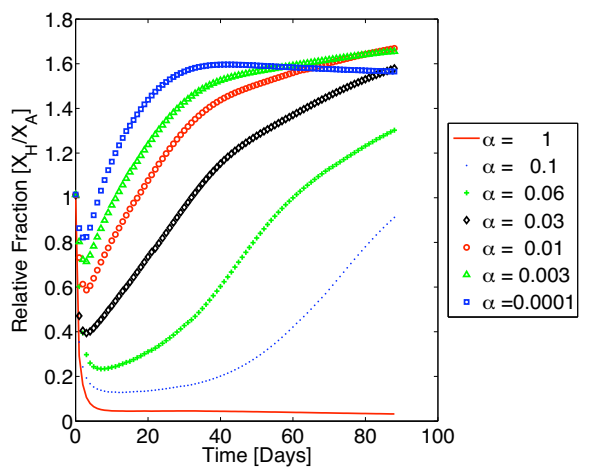

Fig. 5. Variations in $K$ for $\hat{q}_{\mathrm{UAP}}$ and $\hat{q}_{\mathrm{BAP}}$ from Laspidou and Rittmann (2002a) (a) and for COD-based $\hat{q}_{\mathrm{UAP}}$ with scaled $\hat{q}_{\mathrm{BAP}}(\mathrm{b})$.

Plots of relative fraction of heterotrophs to autotrophs as a function of time with varying $K$ and fixed $\hat{q}_{\mathrm{UAP}}$ and $\hat{q}_{\mathrm{BAP}}$. The parameter $\alpha$ scales the values of $K$ from the LR values in Table III multiplicatively, via $K_{\text {used }}=\alpha K_{L R}$. For lower utilization rates (a), low $K$ values are required to yield substantial heterotroph presence in the biofilm; however, when utilization rates are higher (b), even larger $K$ values are able to yield 1:1 or greater species coexistence.

(a)

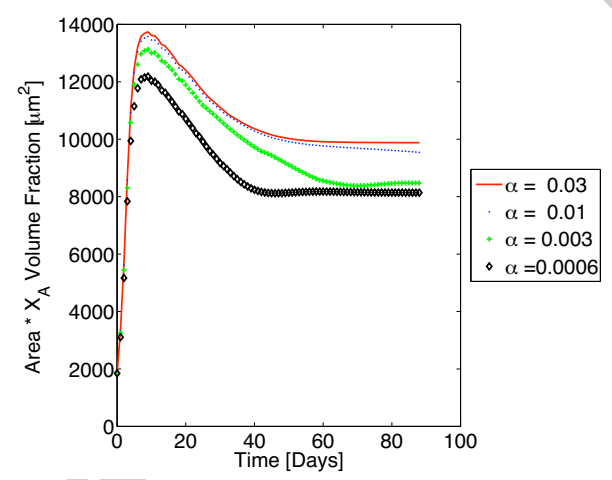

(b)

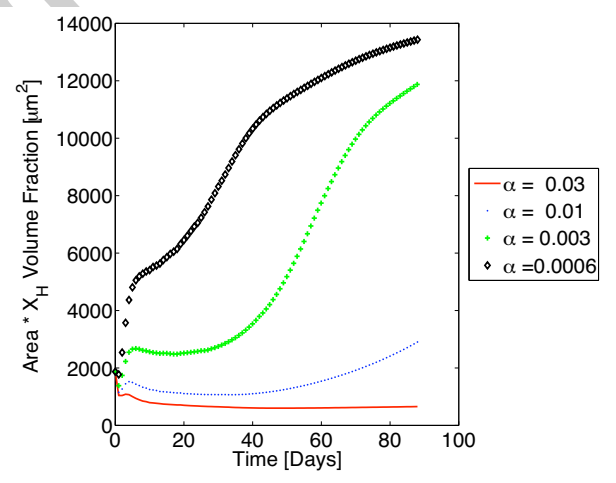

Fig. 6. Plots of autotroph (a) and heterotroph (b) amounts over time.

Shown are curves for a subset of the LR-based $\hat{q}_{U A P}$ cases plotted in Figure $5(\mathrm{a})$. Multiplying the volume fraction of the two active species by the biofilm area at each point in time allows for a comparison of the effect different parameter sets on the total biomass amount. One easily sees from the autotroph plot that SMP consumption has a small effect on the amount of autotrophs in the biofilm, but that the resultant heterotroph amount is more affected. 
(a)

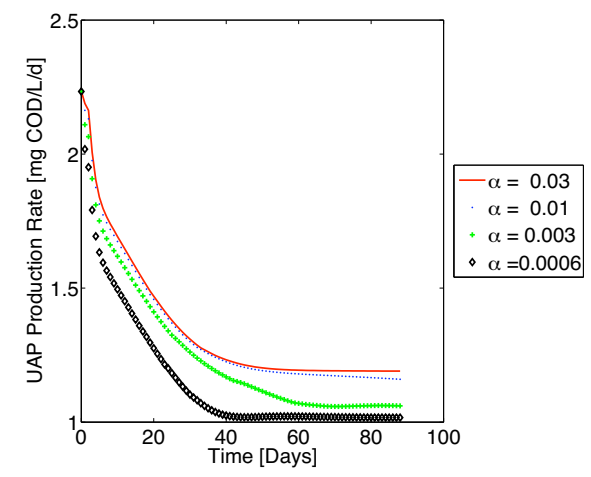

(b)

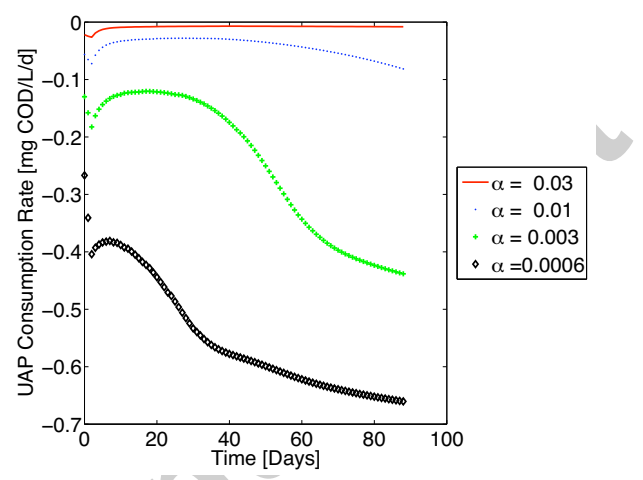

Fig. 7. Plots of (a) UAP production by autotrophs and (b) UAP consumption by heterotrophs over time.

These plots show the trends of UAP production and consumption for the same sets as shown in Figure 6. The UAP production by the autotrophs reaches steady state by about 50 days, which corresponds to the same time period for the autotrophs in Figure 6. In addition, note that the variation in UAP production is quite low, on the order of $20 \%$ for a 2 -orders-of-magnitude variation in $K_{\mathrm{UAP}}$. Consumption of UAP by the heterotrophs shows much more variation with the value of $K_{\mathrm{UAP}}$, and the magnitude of the rate shows exactly why the heterotrophs succeed or fail for the different cases in Figure 6. The time-varying heterotroph amount for the middle $K_{\mathrm{UAP}}$ values in Figure 6 is seen to result from a slowly increasing degradation of UAP (more negative rate). 
(a)

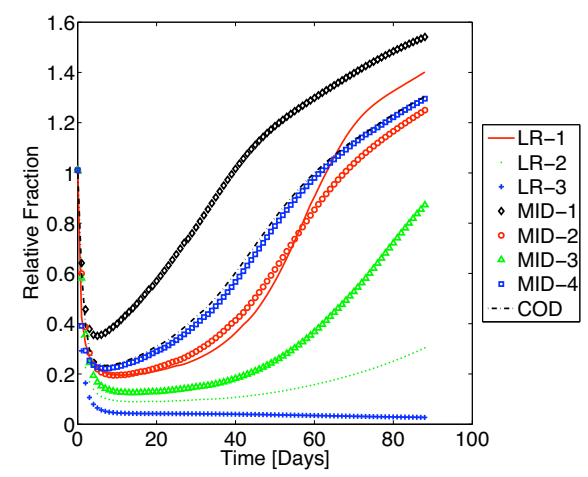

(b)
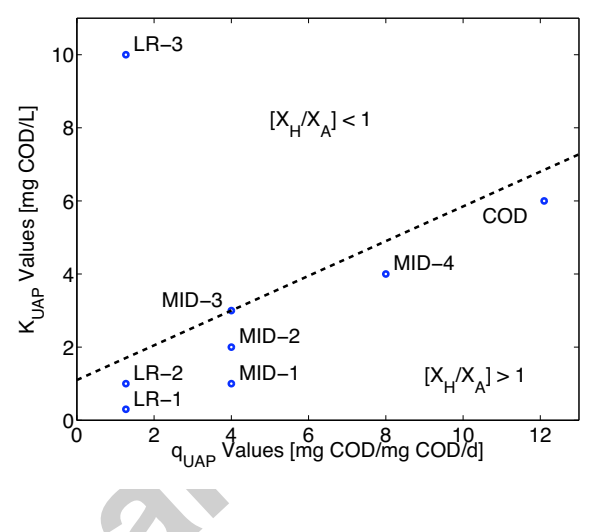

Fig. 8. (a) Comparison of relative volume fractions over time for different test values and (b) a plot of test values in a $K_{\mathrm{UAP}}-\hat{q}_{\mathrm{UAP}}$ space.

(a) This plot shows the relative fraction of heterotrophs to autotrophs as a function of time for the six specific SMP utilization parameter sets listed in Table V. The plot shows that a variety of parameter sets may yield the same or similar biofilm composition. (b) The parameter sets are plotted in a $K_{\mathrm{UAP}}-\hat{q}_{\mathrm{UAP}}$ space with regions labeled based on the relative $X_{h} / X_{a}$ fraction after 90 days of evolution. The dashed line has slope calculated using (10). Any combination of $\hat{q}_{\mathrm{UAP}}$ and $K_{\mathrm{UAP}}$ below the line will allow for heterotroph dominance in the biofilm due to high growth rates, while points above the line will show less heterotroph presence. 

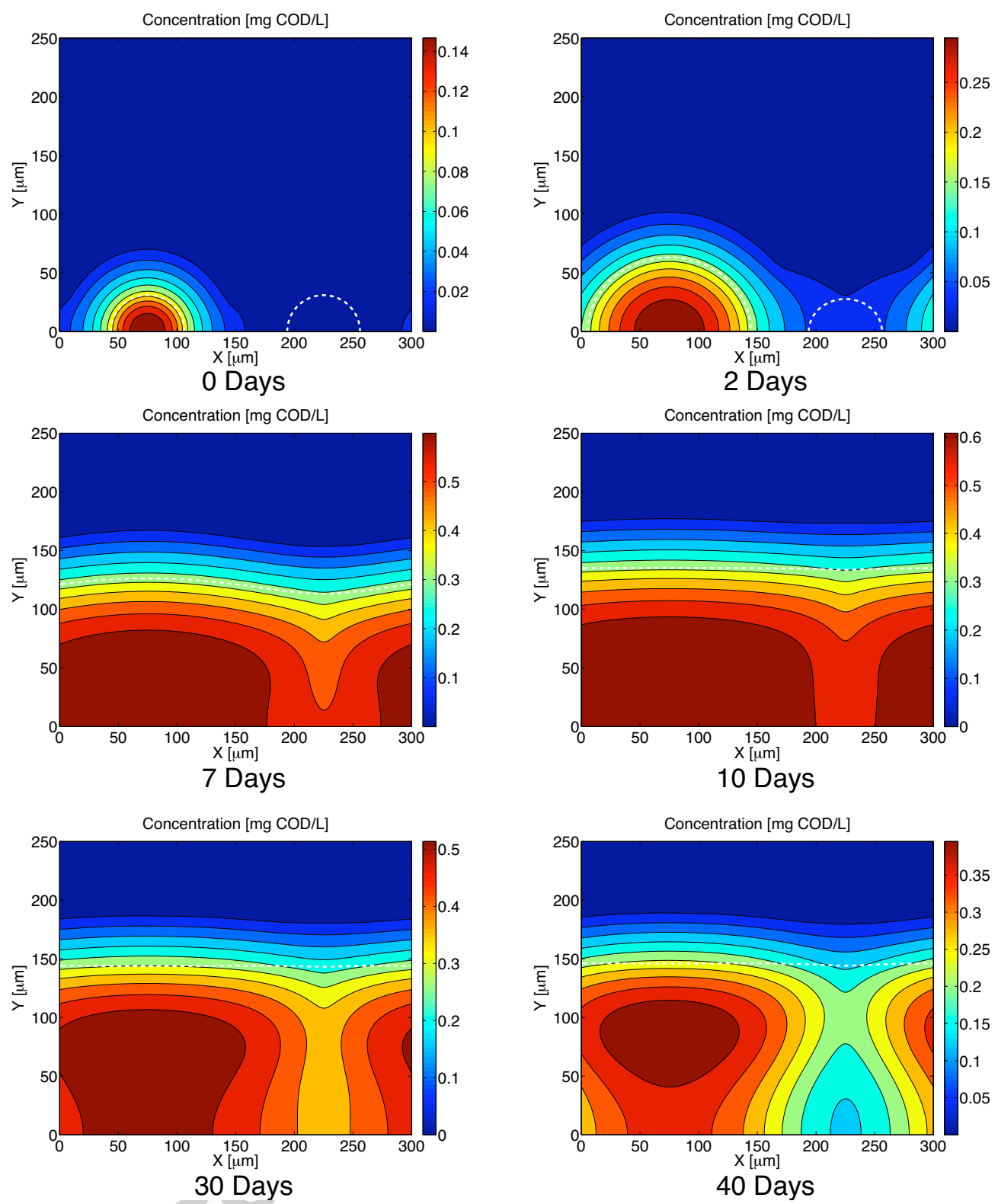

Fig. 9. Time evolution of biofilm interface (white) and UAP concentration.

The biofilm is inoculated with an autotroph (left half of domain) and heterotroph (right half of domain) colony separated by $150 \mu \mathrm{m}$ (5 times the colony radius). Parameter values for this example are from the LR- 1 case in Table V, which is for a $K$-strategist species. Over time the concentration of UAP in the domain first grows and then decreases as the heterotrophs occupy a larger fraction of the biofilm. The peaks in UAP concentration show the highest regions of autotroph activity, while the troughs show the highest regions of heterotroph activity. The biofilm grows flat by 10 days, with the remainder of evolution changing the internal rather than external biofilm structure. 


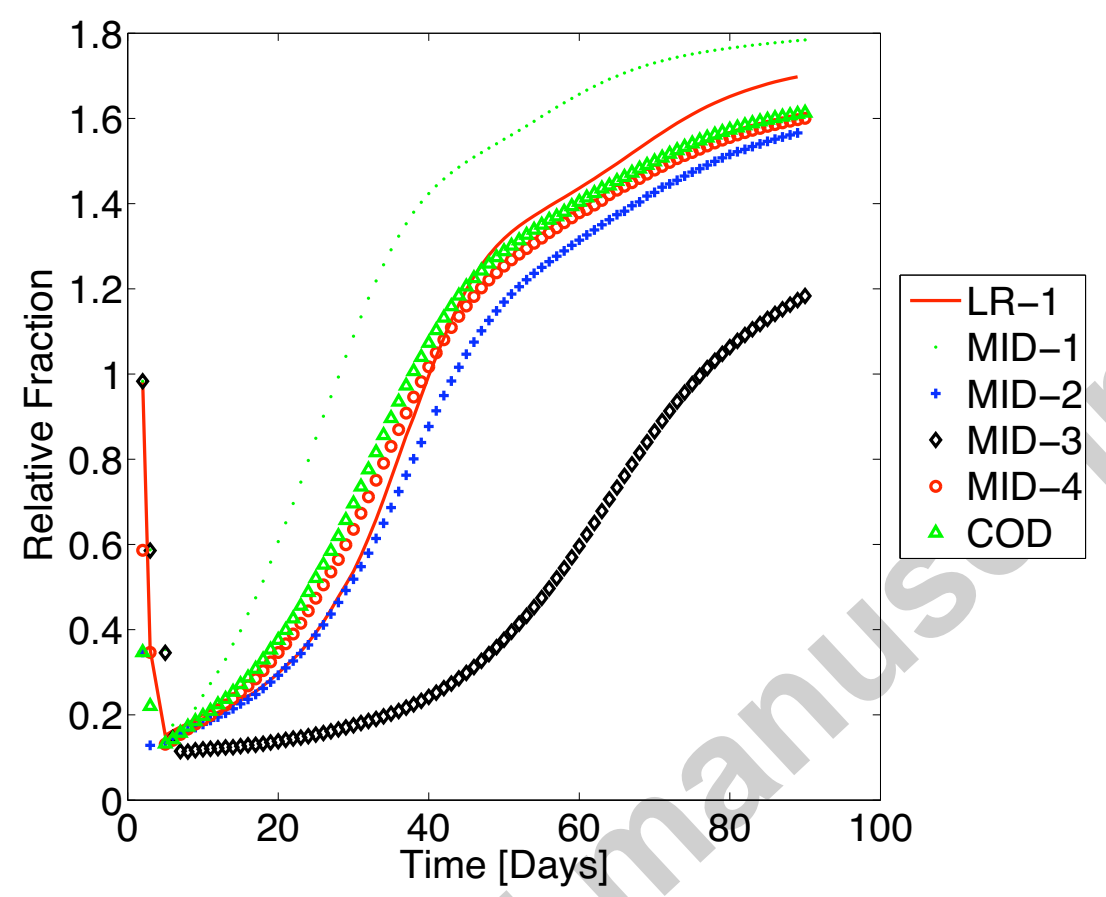

Fig. 10. Comparison of relative volume fractions over time for different test values and a split inoculation.

Similar to Figure 8(a), this plot shows the relative fraction of heterotrophs to autotrophs as a function of time for a subset of the SMP utilization parameter sets listed in Table V. However, in this plot the two species were inoculated apart from one another, as illustrated in Figure 9. Similar to the mixed inoculation, one sees an initial drop in the heterotroph-to-autotroph fraction, and soon after a recovery by the heterotrophs; this recovery is delayed or may even be prevented with a larger colony separation distance. Note that the split inoculation yields a higher long-term heterotroph-to-autotroph ratio than the mixed case (Figure 8(a)), which is a result of the decreased competition for space in the biofilm. 


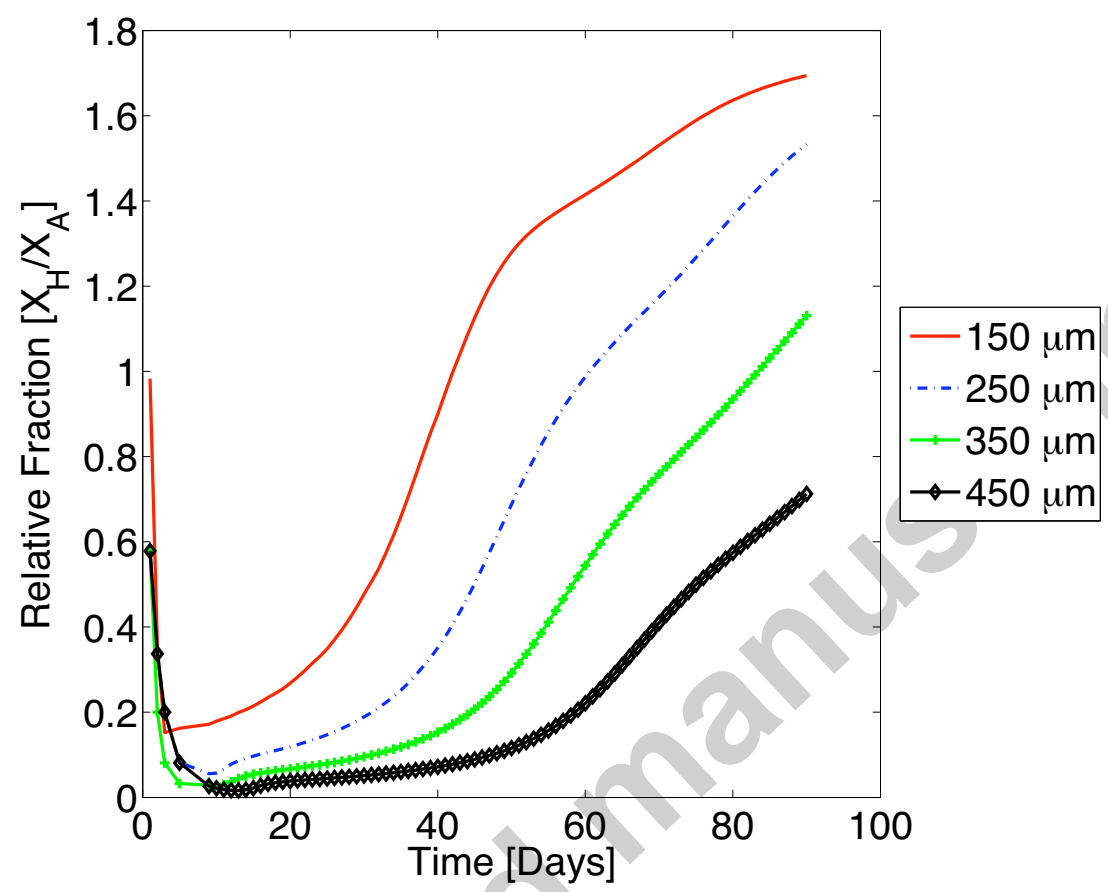

Fig. 11. Time series of relative heterotroph fraction for different inoculation distances.

Two semi-circular colonies were inoculated with their centers separated by different distances. In all cases the parameter set used was LR-1 from Table V. The fraction drops relatively quickly for all cases because the heterotrophs are separated from their SMP source, but as the autotroph colony grows then the heterotrophs are provided SMP electron donor. When the initial colony separation is increased, the time until heterotroph growth is also increased. 


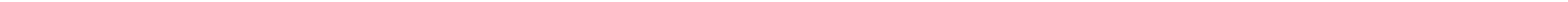




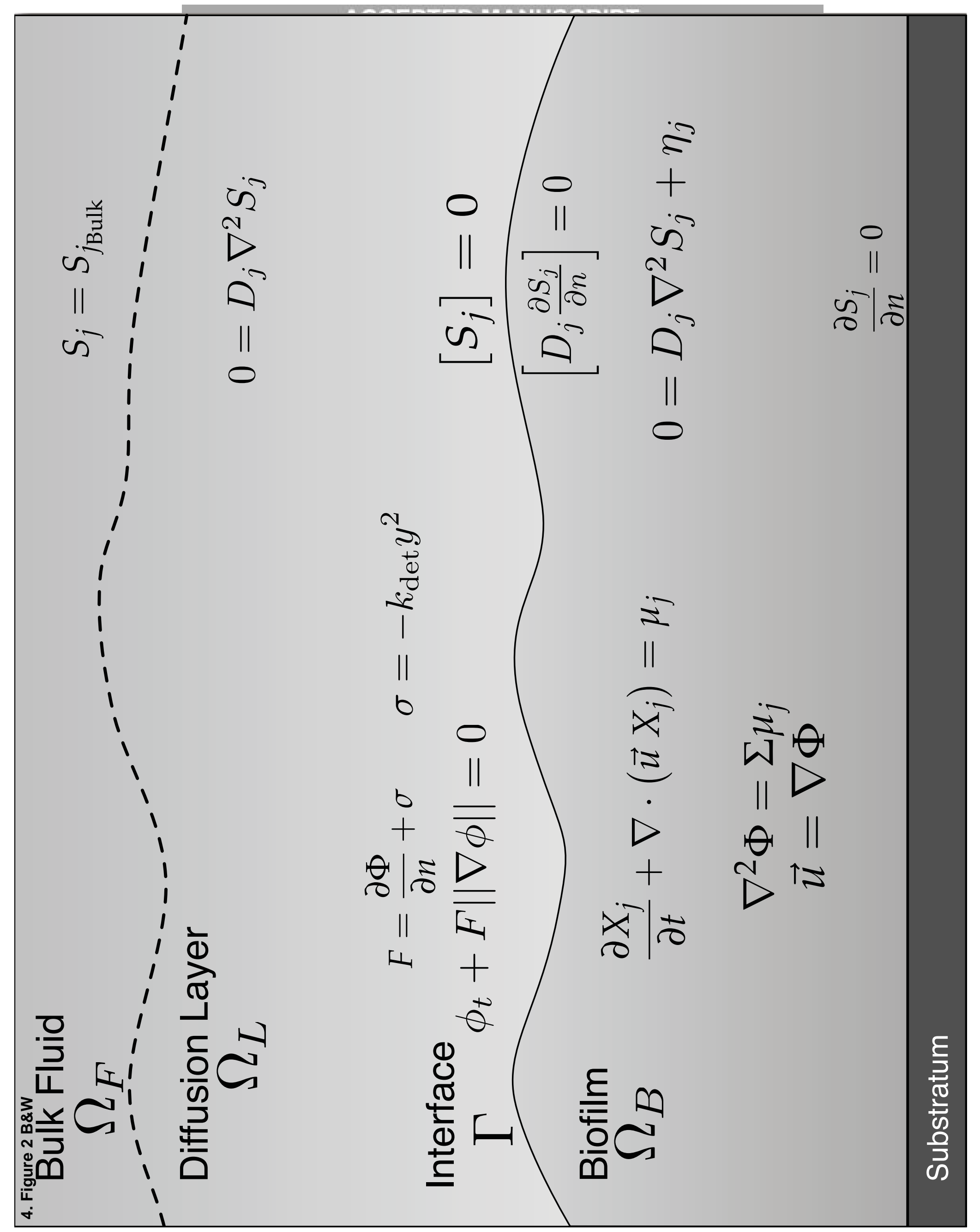




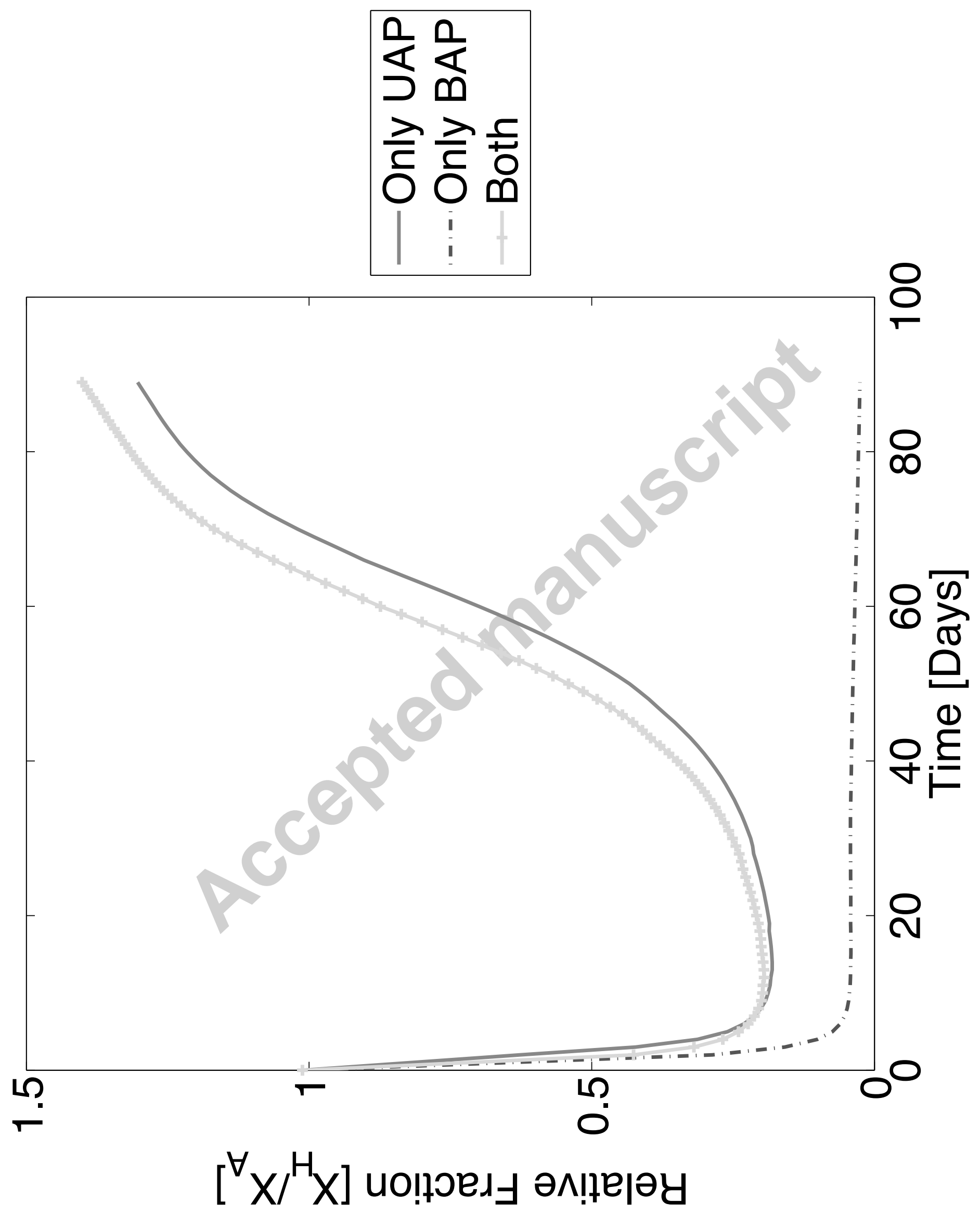




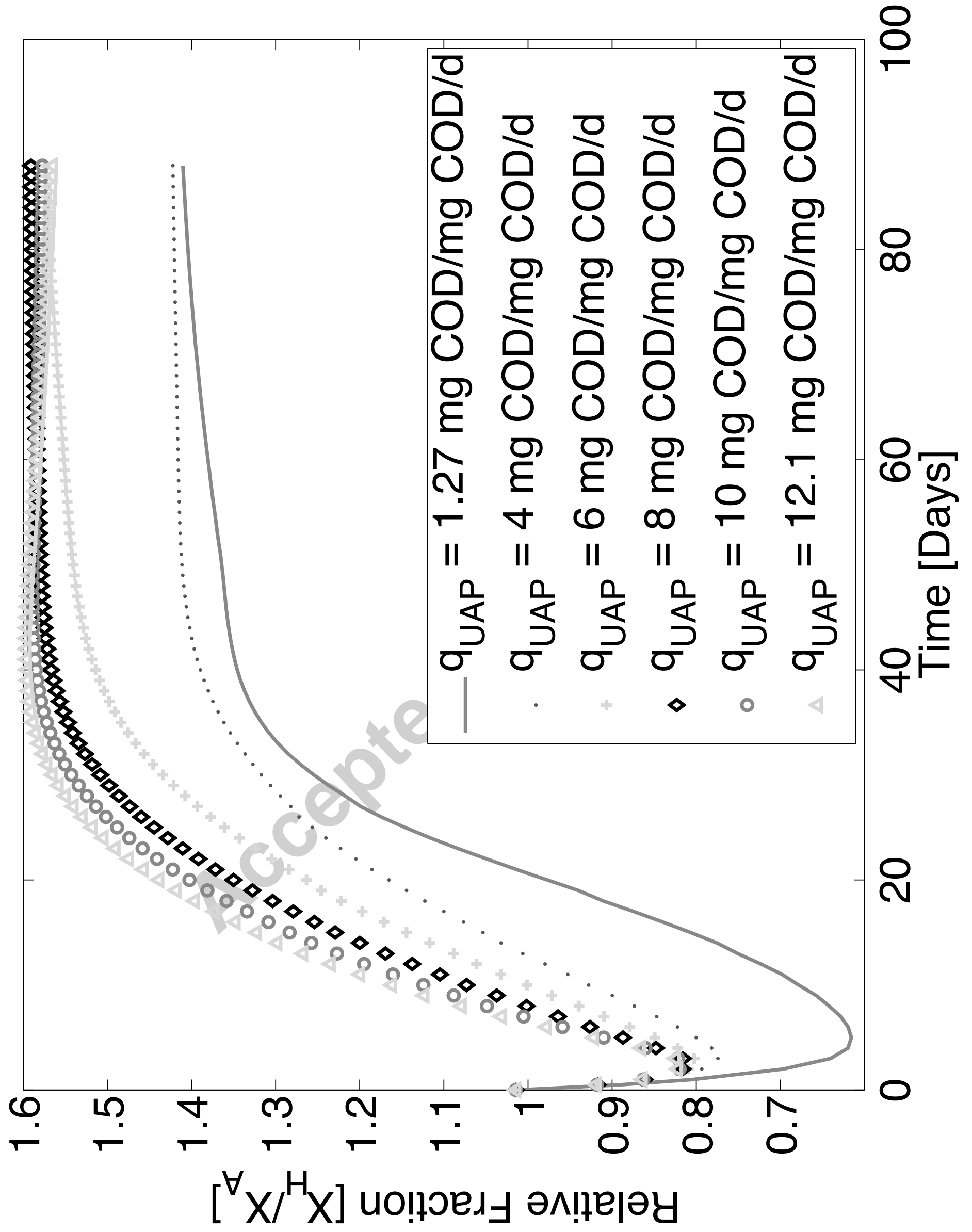



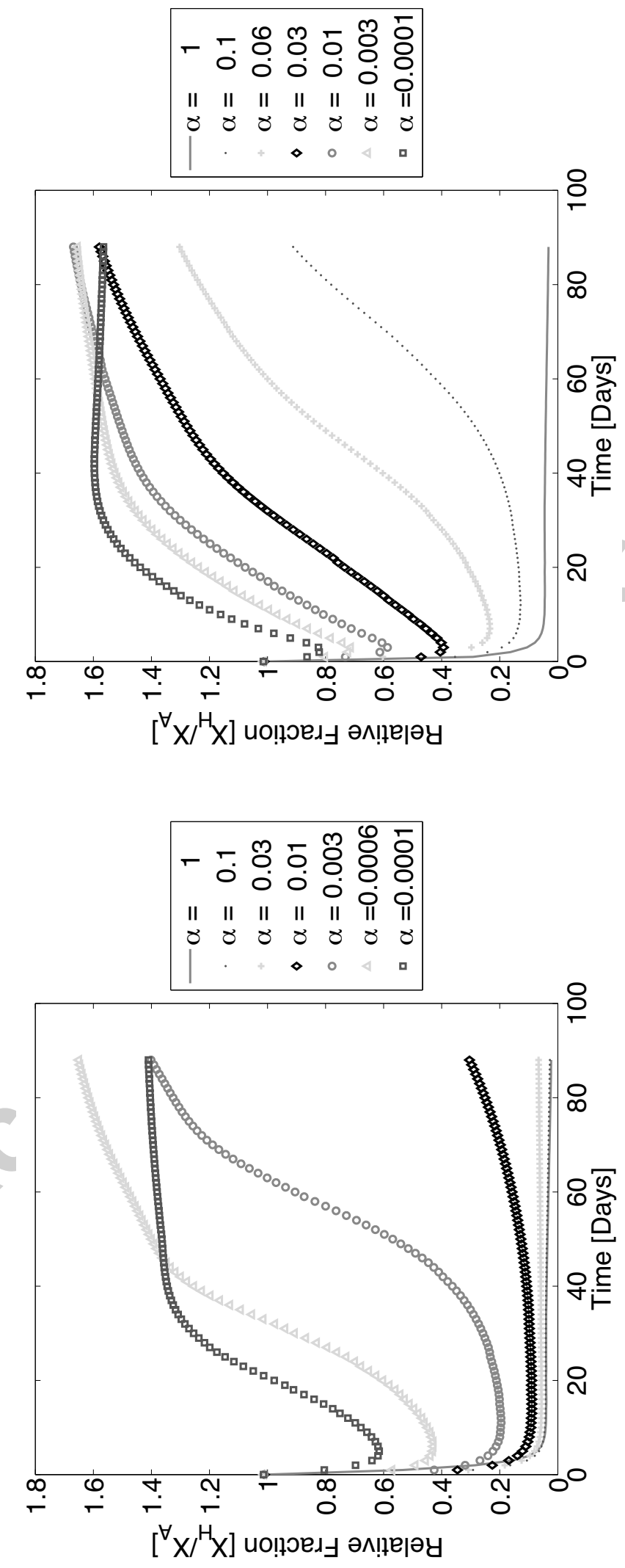

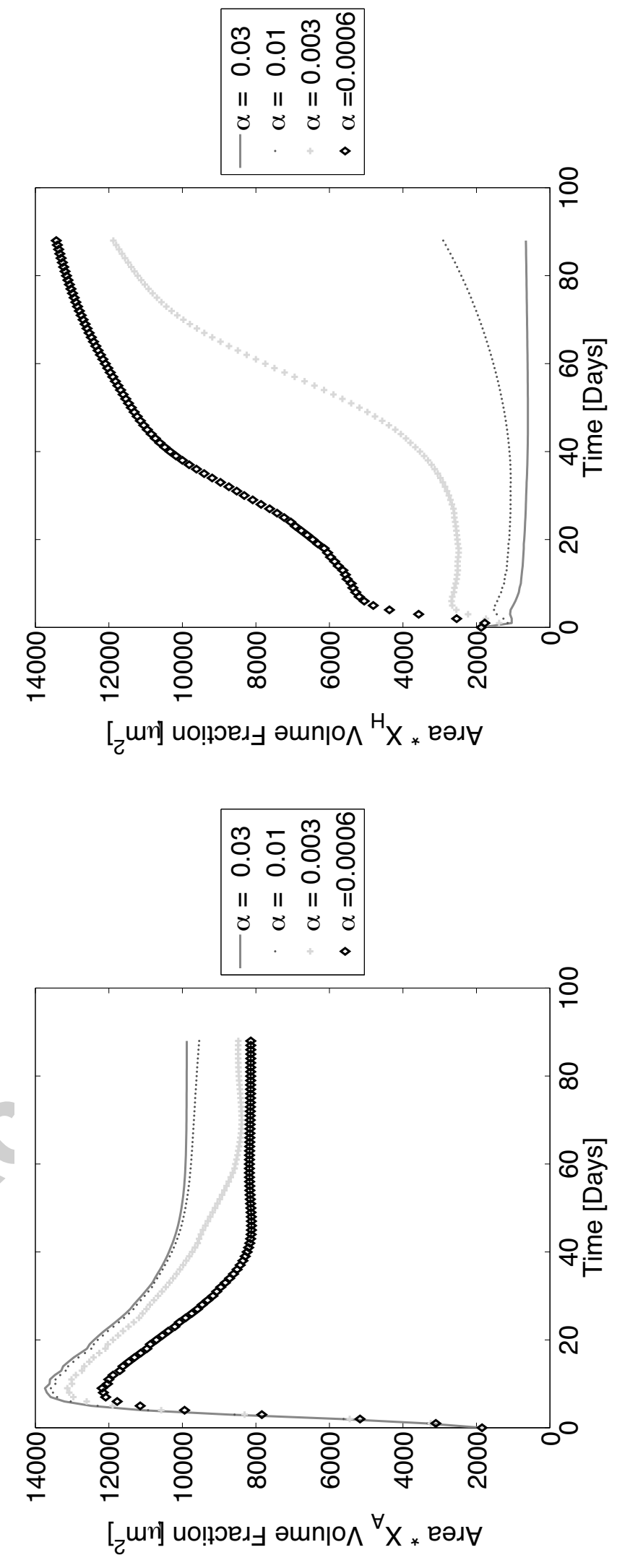

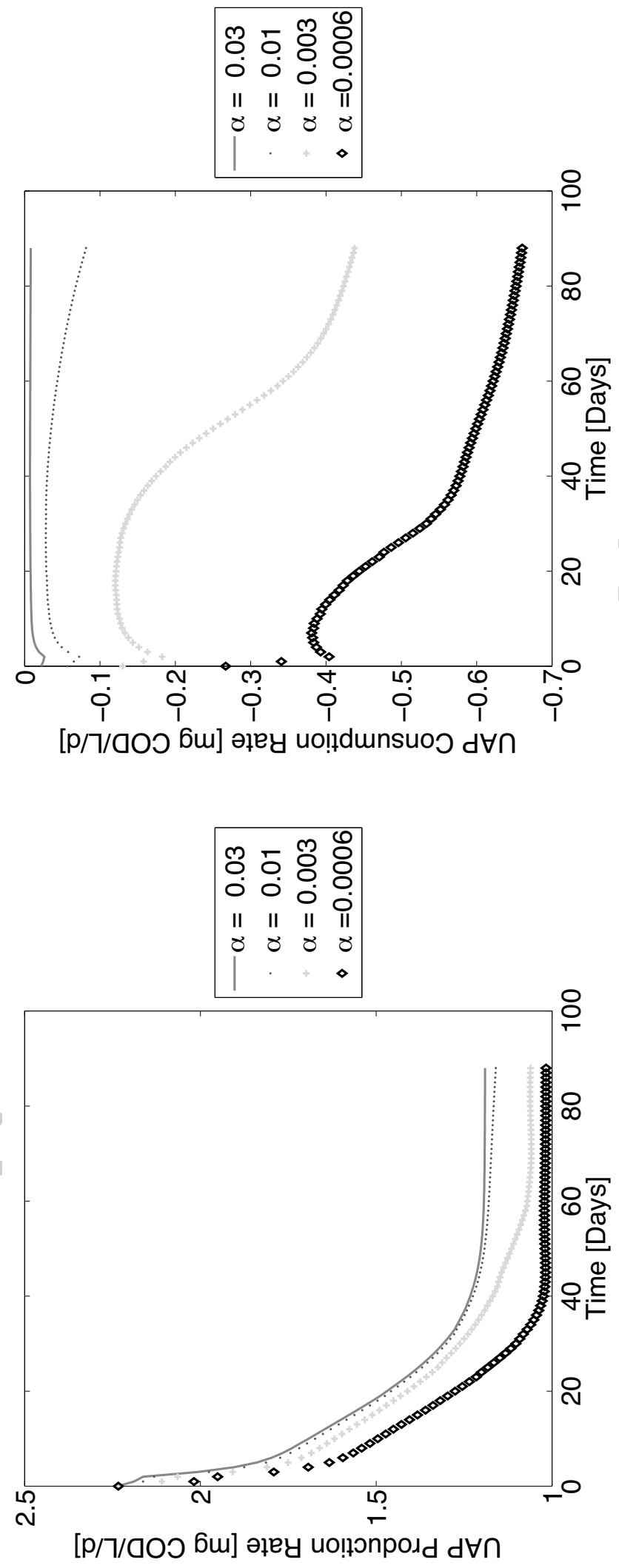

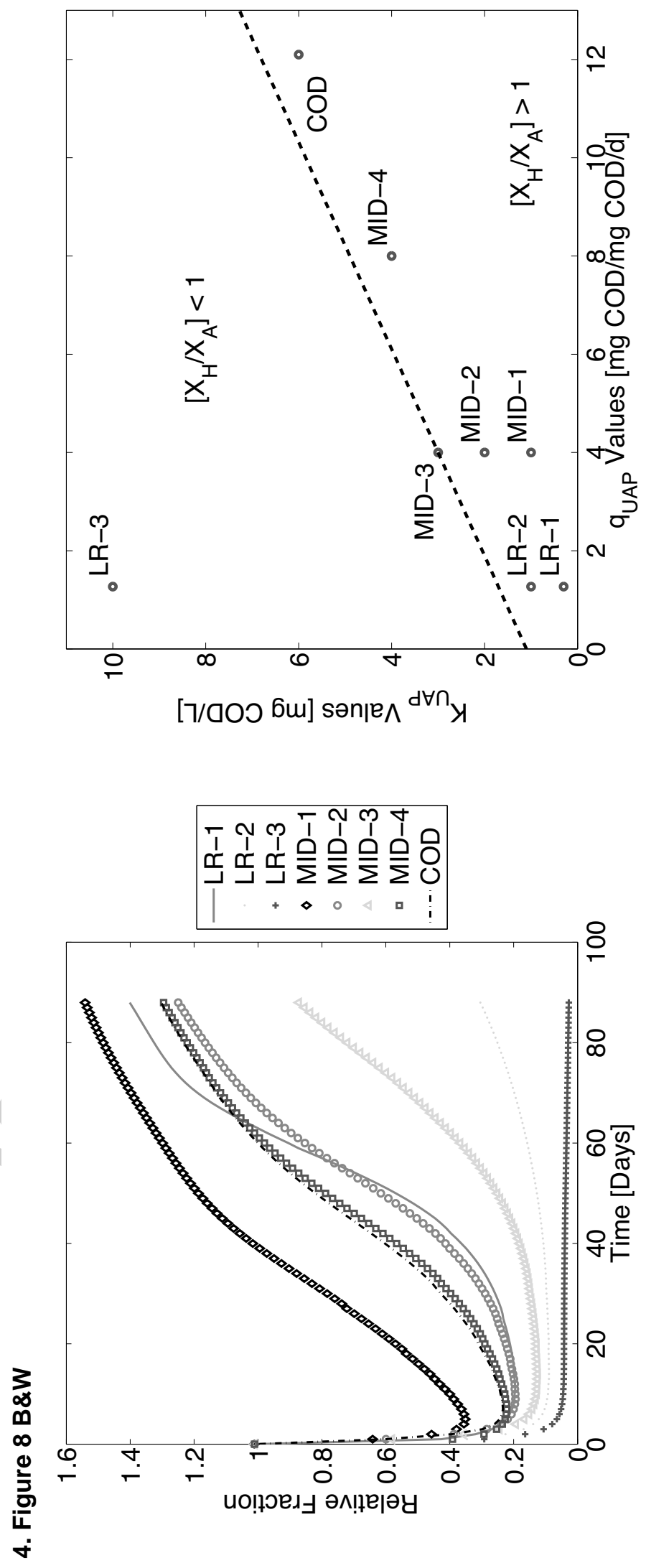
4. Figure 9 B\&W
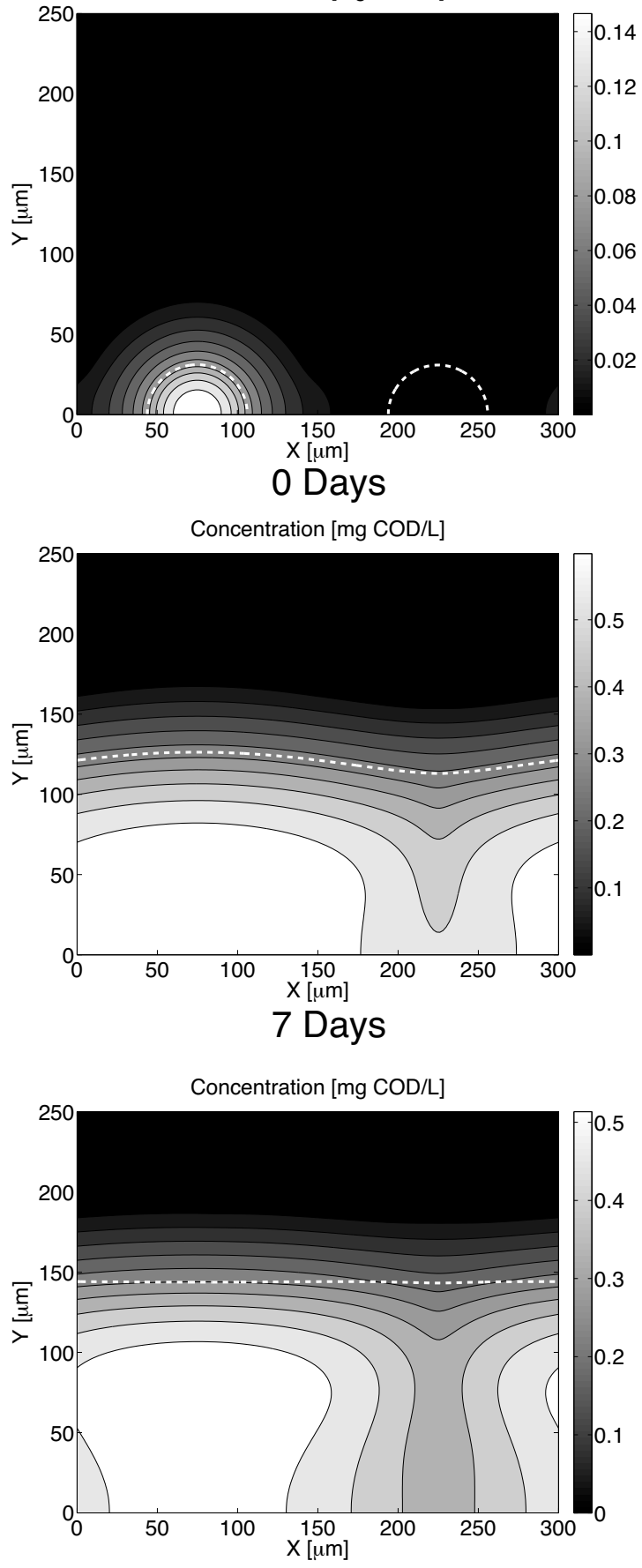

30 Days
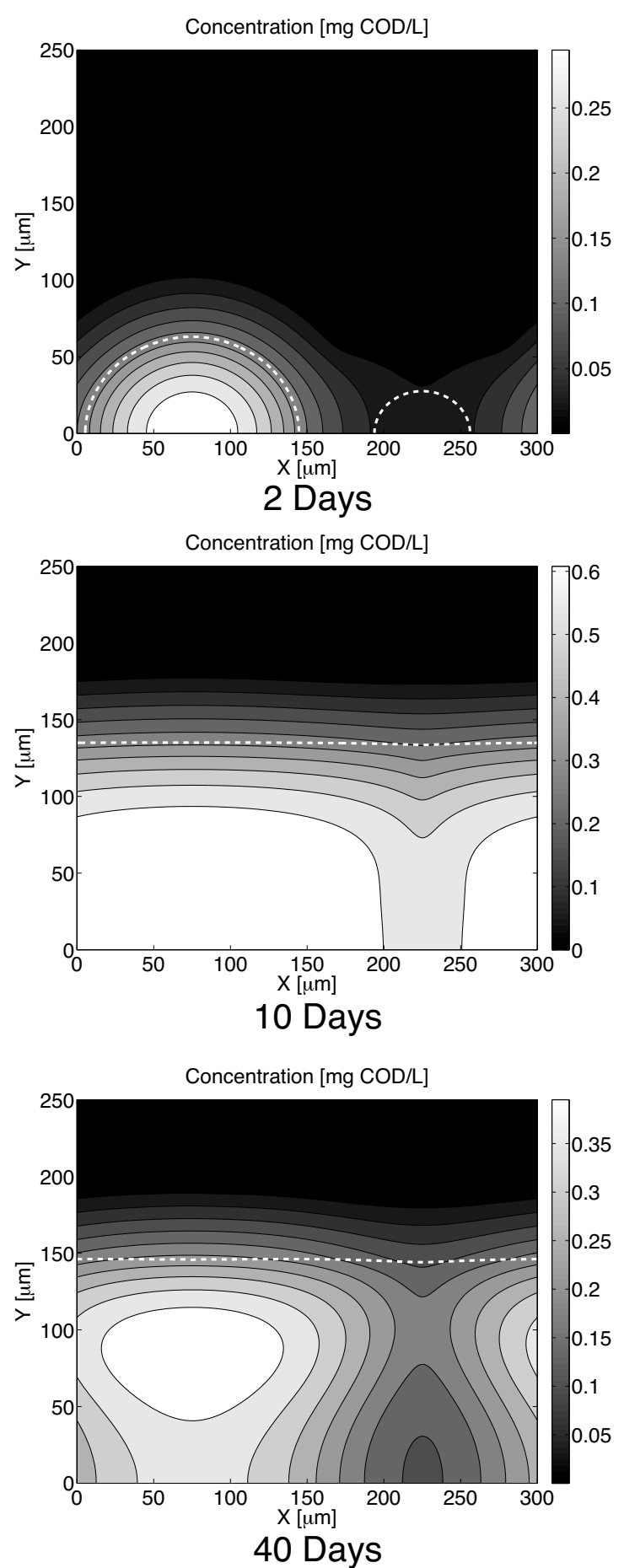

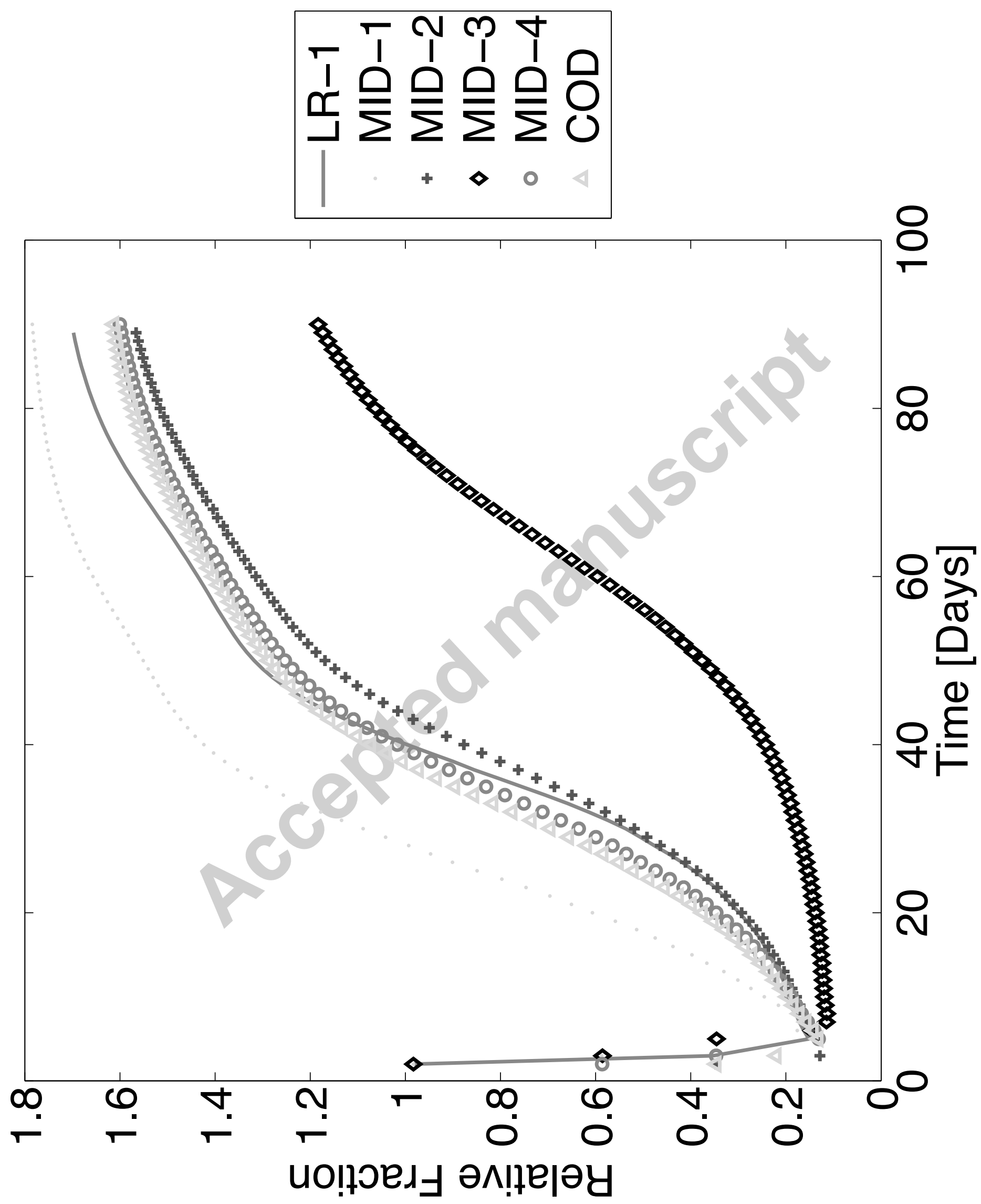


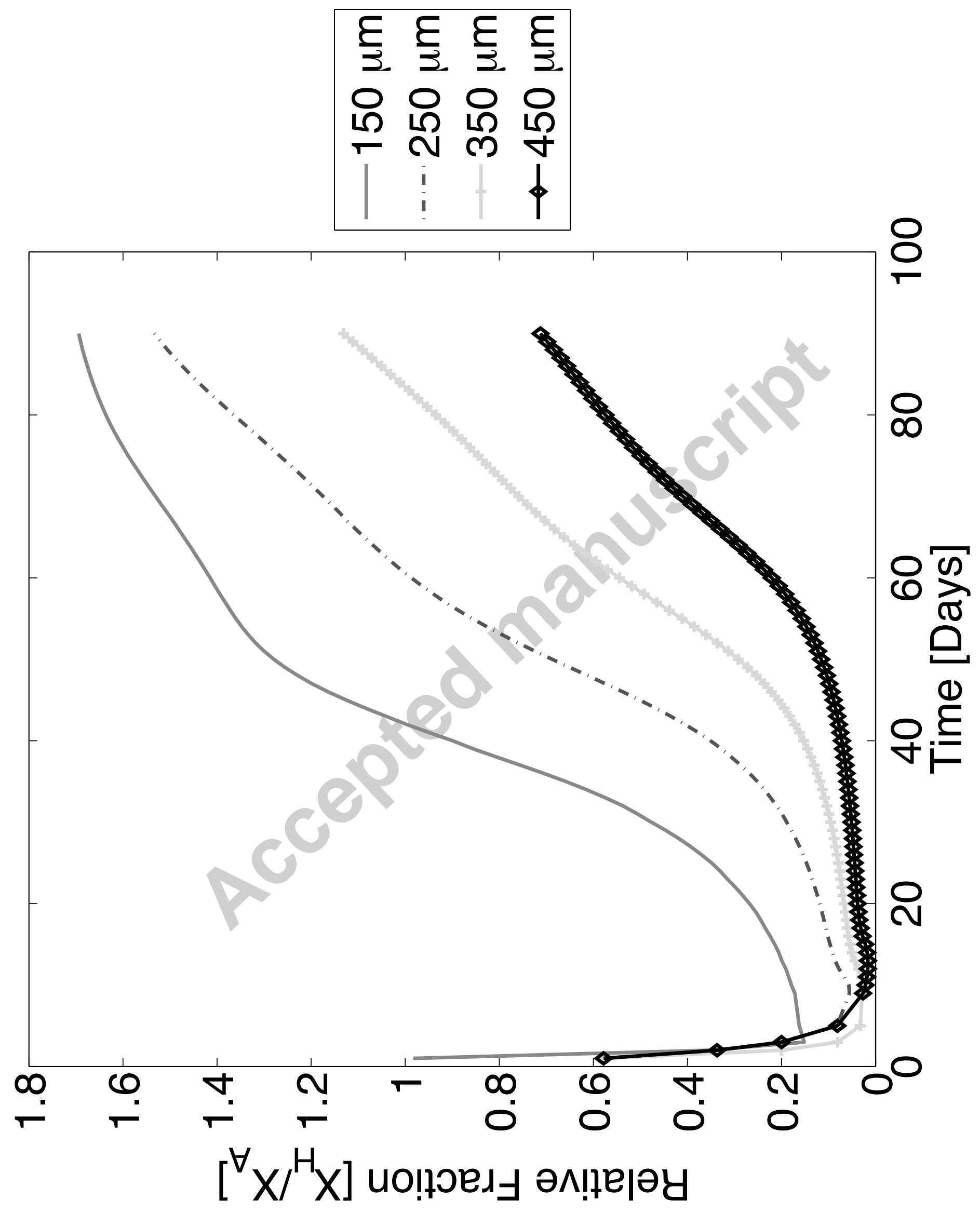

